\title{
Microscopic simulation based uncontrolled intersection performance assessment
}

\author{
Suprabeet Datta ${ }^{1}$ ] . Siddhartha Rokade ${ }^{1}$ (D) Sarvesh P. S. Rajput ${ }^{1}$ (I)
}

Received: 16 June 2020 / Accepted: 14 September 2020 / Published online: 24 September 2020

(c) Springer Nature Switzerland AG 2020

\begin{abstract}
Performance of an urban corridor comprising of urban streets, signalized and unsignalized intersections gets highly influenced by the level of traffic operations at the unsignalized intersections. Unsignalized intersections under mixed traffic behave in uncontrolled manner due to lack of stop or yield signage. This study aims at assessing performance of urban uncontrolled intersections. Number of conflicting vehicles and their flow rates were used to develop mathematical models for service delay. Field service delays from 13 selected intersections form India were estimated and validated using PARAMICS after calibration using real time video data. Mean service delay for turning movements are analysed based on microscopic field investigation of driver travel time under different driver behavior. Two different traffic operational scenarios considering four-wheeler SUV stream at three-legged and four-legged intersections are compared. A thorough statistical investigation indicated that the conflict technique can predict $52 \%$ of field estimated service delays with a sensitivity of as low as $3 \mathrm{~s} /$ vehicle/lane for various traffic streams. The service delay, driver turning time and queue length models suggested can be utilized by practicing engineers to estimate intersection capacity and level of service of turning movements for better traffic operational management.
\end{abstract}

Keywords Conflicting flow rate $\cdot$ Turning time $\cdot$ Service delay $\cdot$ Microsimulation · Uncontrolled intersections · Queue length

\section{Introduction}

Uncontrolled intersections provide access to drivers from one road to the other, having varied bordering land use without following priority rules at their ease. Movements at these junctions are not governed by signal lights and thus provide alternate access to signalized intersection right-turning drivers in the absence of free-right at such facilities. Figure 1 shows a typical snapshot of the right turning movement at uncontrolled intersections in urban India. Turning movements are feasible only when suitable gaps are available in the opposing traffic and get delayed if adequate gaps are void. Therefore, turning vehicles cause delay to conflicting vehicles in the through traffic stream, which in turn reduce speed leading to unnecessary blockages or bottlenecks at turning points. The creation of jams decreases capacity increasing volume to capacity ratio and queue lengths. Under extreme cases, when delay to the turning drivers goes over a certain threshold, the driver gets exasperated (i.e., waiting time increases), resulting in forced gap entry. Forced gap acceptance at uncontrolled intersections often leads to rear-end collisions. TRB [1] and IRC [2] use the term "control delay" for stop-controlled and unsignalized intersection, which is the total delay

Electronic supplementary material The online version of this article (https://doi.org/10.1007/s42452-020-03509-0) contains supplementary material, which is available to authorized users.

Suprabeet Datta, 144040009@iitb.ac.in; Siddhartha Rokade, siddhartharokade@gmail.com; Sarvesh P. S. Rajput, spsrajput1976@gmail.com | 1 Department of Civil Engineering, MANIT Bhopal, Bhopal, Madhya Pradesh 462003, India. 


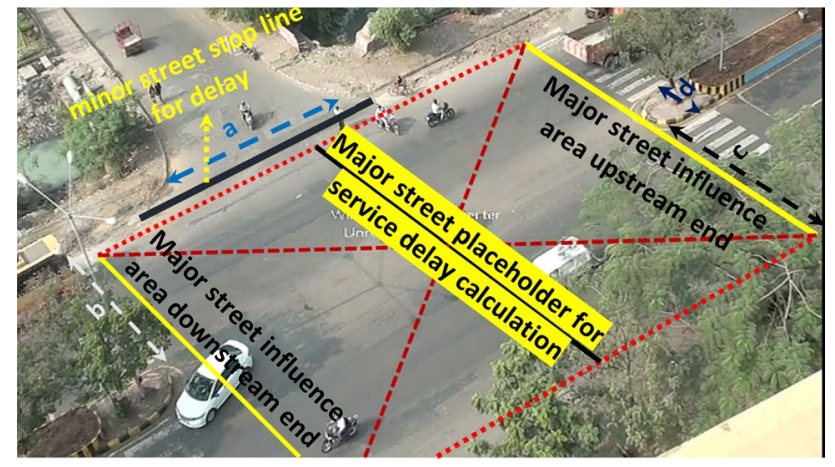

Fig. 1 Screenshot of typical three-legged uncontrolled intersection showing influence lines used for service delay, driver turning time and conflicting flow rate calculations for the present study

experienced by a driver at the stop line while accepting a gap due to any type of control.

Preliminary investigation on delay models for minor roads was proposed by Daganzo [3] and Kimber et al. [4] based on intensity of traffic flow (ratio of volume to capacity) at unsignalized intersections. Degree of saturation was considered for priority controlled intersection minor road delay assessment by Hiedmann and Wegmann [5]. The complex exponential relationship between traffic flow rate and service delay was replaced with generalized delay model based on traffic simulation by Tian et al. [6]. Lutinnen [7] related traffic volume and delay for unsignalized intersections. Chodur [8] investigated other parameters effects on capacity models while assessing delay. Methodological assessment of unsignalized intersection stopped time delays was performed by Pitzinger and Spacek [9]. The authors invented some complex calculations based on the additive conflict flow (ACF) technique based on subjective repurcations applied to intersections with varied geometric attributes. Studies like $[10,11]$ suggest that delay is an essential parameter for performance and safety assessment of uncontrolled intersections. Merging behavior of right turning vehicles were studied microscopically at T-intersections by Kanagaraj et al. [12]. The authors developed merging behavior models considering forced and normal merging process and validated their model with field estimated results. Forbush [13] established a real-time automated delay estimation technique for through lanes at signalized intersections. The author neglected the effect of shared lanes on multiple-modes which is considered in this research. Gap acceptance behavior at median turnarounds within the city of Jordan was studied by Obaidat and Elayan [14]. The researchers concluded that female drivers accept longer gaps compared to male ones and that older drivers accept longer gaps than younger ones. The authors also inferred that drivers having longer waiting times take shorter gaps. Caliendo [15] applied the negative binomial (NB) distribution to study minor road service delays under three different traffic operational scenarios of unsignalized intersections using micro-simulation in AIMSUN. The authors formed three logistic regression models relating peak hourly entry demand volume and conflicting flow rate as explantories to average delay, stopped time and maximum queue length. Menendez et al. [16] developed delay equations that can be applied to assess uncontrolled roudabouts involving two or three conflicting $[\mathrm{C}]$ or non-conflicting streams $[\mathrm{NC}]$ and validated with the HCM [17] control delay model in Zurich, Switzerland. Sil et al. [18] studied influence of service delay (SD) on U-turn merging time (MT) for five vehicle categories considering conventional and forced merging situations based on time-distance plots. The authors also studied the effects of opposing traffic flow on U-turn merging time at uncontrolled median openings. Swift and gradual merging were found to significantly different from each other under mixed traffic. Guller and Menendez [18] determined delay of priority and non-priority conflicting streams for five uncontrolled traffic facilities (4 nos. intersections and 1 no. roundabout) consisting of pedestrian and tram movements. The authors developed equations for capacity of conflicting [C] and non-conflicting [NC] streams using ACF and used these to estimate delay based on flow ratio (Y). A comparison with the HCM [17] delay values suggested that the model showed a maximum of $3 \%$ error with as low as $0.1 \%$. Also, the method was able to detect streams having larger delays with a minimum sensitivity of $4 \mathrm{~s} /$ vehicle. Low velocity of turning drivers and complex encroachment perspectives due to through or conflicting flow makes delay assessment under heterogeneous traffic flow conditions more critical. Shatnawi et al. [19] developed a vehicle delay estimation algorithm based on automatic measurement of real-time data from detectors and signal controllers at intersections. The outputs from the proposed algorithm was statistically validated and tested using microscopic simulation (VISSIM) based on initial queue size under varying turning volume percentages. The model proved to be reliable and effective under assorted signal timing situations. Studies on delay assessment of stop and yield controlled intersections are plenty [20-25]. Sun and Elefteriadou [26] developed urban lane-changing models in CORSIM based on two experiments namely, a focus group study and an in-vehicle driving simulator test. The authors developed attributes for lane changing and gap acceptance criteria. The authors utilised the proposed framework as a run-time extension (RTE) plug-in in CORSIM architecture after aggregating selective driver behavioural paramters within their simulation model. The traffic facility tested was an urban arterial. Comparison between the existing CORSIM model and the newly developed model indicated that the authors 
successfully addressed lane changing behavior even under varied congestion levels. Sun, Zhang and Chen [27] carried out comparative analysis between VISSIM and CORSIM for urban road networks consisting of intersections in Shanghai, China. The measure of effectivenesses based on which the comparison was made are usability of software, average control delay, mean queue length and crosssectional traffic demand volume. The scales of comparison were carried out for each software depending upon the level of congestion and intersection geometric attributes. The authors found out that driver behavior, traffic environment settings, vehicle types are the main calibration paramters which influences the comparison. Finally, the authors suggested utilization of microsimulation software (CORSIM, VISSIM or PARAMICS) depends primarily on the type of intersection and the saturation flow levels during peak hours. It is interesting to note that very few studies are rampant on service delay assessment of multi-modal uncontrolled intersections under non-lane based traffic environments [28]. Through this study, the researchers would like to address microscopic assessment of service delay, driver turning time (both right and left) and queue length (major and minor) for various motorized vehicle categories at multi-modal unconventional uncontrolled intersections under mixed traffic. Service delay estimated using the field microscopic technique is validated using the Quadstone PARAMICS [21] microsimulation software. The tetra-pleat objectives for this research are:

1. Development of regression models based on the microscopic analysis relating effect of number of conflicting vehicles and conflicting flow rate on field estimated values of service delay and driver turning time.

2. Development of conflict technique based service delay and queue length models at multi-modal intersections (involving shared PT lines) based on "SUV" stream assessment.

3. Identification of performance parameters affecting service delay of uncontrolled intersections through microscopic simulation. Validation of the microsimulation outputs for the field estimated data under two different operational scenario.

4. Generalized equations for service delay, driver turning time and queue length formulation using logistic regression.

This research is structured in seven sections. The first section of this paper portrays a brief background of the research carried out along with recent studies on service delay assessment, microscopic simulation based delay estimation. The first section also enlists the tetra-pleat objectives of the study. The second section gives a detailed discussion on the intersections selected along with the data collection or the data extraction strategy towards service delay, driver turning time and conflicting volume (flow rates) estimation. The third section attracts the readers attention towards development of empirical relations service delay and driver turning time with number of conflicting vehicles and their flow rates. The fourth section shows minor/major road service delay and queue length estimation procedure based on the "Additive conflict technique" approach [12] for various traffic streams. This section also shows application of the proposed conflict technique based approach to evaluate two traffic operational scenarios (Type-I three legged intersection with shared public transit lanes and Type-II four-legged intersection with shared public transit lanes) based on priority rules followed under mixed traffic. The fifth section shows validation of the proposed "conflict technique" approach for service delay and queue length estimation after calibrating all the 13 selected intersections in PARAMICS for both morning and evening peak traffic flows. The sixth section shows statistical modelling of service delay, driver turning time and queue length (all of which influence uncontrolled intersections performance) based on the results obtained from the PARAMICS assuming a negative exponential distribution of headways. Three equations for service delay, driver turning time and queue length were finally presented considering turning demand volume, conflicting flow rate and number of conflicting vehicles as explantories based on marginal effects. The last section briefly summarizes the study conducted and carefully concludes the research.

\section{Study Area, Data Collection and Extraction}

Thirteen uncontrolled intersections (both 3-legged and 4-legged) are selected from northern, eastern, western, and central parts of India. The sites are free from extensive roadside frictional activities like on-street parking, unsynchronized pedestrian and NMT activity, or presence of public transit stops. Detailed intersection geometry after manual inventory for all the selected sites is represented in Table S1. ${ }^{1}$ Data collection at the selected uncontrolled intersection sites is performed using the video-graphic technique for which high definition cameras are installed at appropriate vantage points (on top of buildings and over median spaces of the major street) to capture the turning movements covering the entire conflicting activities within the intersection influence area. Data is collected for typical 5 to $7 \mathrm{~h}$ within weekdays comprising of

\footnotetext{
1 Table S1 provided along with Supplementary material 1.
} 
both morning and evening peak periods. The converted and filtered videos are operated in the laboratory using a suitable video editing software for creating placeholders after drawing lines on the video. Table $S 2^{2}$ shows the turning and major/minor street vehicular compositions during the peak periods used for the study. The thirteen intersections belong to eight cities (namely, Kolhapur, Vashi, Indore, Bhopal, Moradabad, Lucknow, Durgapur, and Kolkata) from four states of Maharashtra (Kolhapur and Vashi), Madhya Pradesh (Indore and Bhopal), Uttar Pradesh (Moradabad and Lucknow) and West Bengal (Durgapur and Kolkata). Reference line measurement and placeholder identification is most important for service delay estimation. The stop line bar functions as the reference placeholder for service delay measurement at stop or yield controlled intersections. But, for the present study, the major street placeholder and a fictitious minor street stop line bar are drawn on the videos, which will function as reference lines for service delay measurement, as shown in Fig. 1. Service time delay for turning vehicles is the time difference between vehicle front wheel arrival and the rear-wheel departure from the imaginary reference line and the placeholder. The turning driver trajectory under heterogeneous flow conditions with poor consistency is not perfectly parabolic. As a result of which, more than $90 \%$ of right and left turning movements interfere with the conflicting traffic. Turning drivers never follow priority rules at uncontrolled intersections and involve themselves in the forced entry without stopping at the reference line making situations risky. Thus, the reference line and the placeholder shown in Fig. 1 is dynamic and virtual. The delay during turning service to a vehicle is measured from arrival time $\left(T_{1}\right)$ of the front wheel at the virtual line to the departure time $\left(T_{S}\right)$ of the rear wheel over the virtual line. Fig. $\mathrm{S}^{3}$ shows service delay data extraction through screenshot imaging. In this research, service delay is determined microscopically with the help of conflicting flow for a subject turning vehicle. Let us assume that $T_{1}$ is the time of arrival of the subject turning at the reference placeholder, $T_{S}$ is the time of departure of the subject turning vehicle, $N_{\mathrm{S}}$ be the number of observed conflicting or through traffic vehicles for the subject vehicle which also includes the conflicting vehicle passing just after the departure of the subject turning vehicles and $T_{i}$ be the time of arrival of the ith conflicting vehicle at the reference placeholder. Conflicting flow rate for subject turning vehicle is obtained using Eq. (1).

\footnotetext{
2 Table S2 provided along with Supplementary material 1.

${ }^{3}$ Fig. S1 provided along with Supplementary material 2.
}

Conflicting flow rate $=\frac{N_{s}}{T_{i}-T_{1}}$

A maximum accepted gap of $10 \mathrm{~s}$ is used based on intersection sight distance and approach speed criteria, as suggested by Kittleson and Vandehey [28]. During off-peak periods when traffic volume is less, the conflicting vehicles often use the last few seconds of the accepted gaps to clear the intersection influence area after the subject turning vehicle has departed. Therefore, if $T_{i}-T_{S}$ is more than $10 \mathrm{~s}$, then the conflicting vehicle using the last few seconds of the accepted gaps is abandoned from the dataset. Driver turning time for right and left-turning vehicles is determined in this study. Turning time was measured as the time gap between rear wheelbase passage over the reference placeholder $\left(T_{S}\right)$ and back wheelbase passage over the turning line. The turning line (White discontinuous marker), as shown in Fig. S1 (see footnote 3 ) is another virtual reference placeholder where the right or left-turning vehicles complete their turning maneuver and merges with the conflicting traffic flow. The turning line where the drivers combine with the conflicting/through traffic flow is dynamic and depends upon the turning vehicle dimension and mean position of the turning line as observed for the sites. The position of turning line at the downstream end of the intersection influence area is depicted in Table S3. ${ }^{4}$

\section{Empirical Relationships for field estimated service delay and driver turning time}

Heavy volume of service delay $\left(D_{\text {service }}\right)$ and conflicting traffic vehicle $\left(N_{S}\right)$ for right and left-turning vehicles are developed using the microscopic analysis presented through this research. The effects of the opposing and through traffic flow rates on the turning vehicle service delay is studied. The turning time required by right and left-turning drivers for several vehicle classes is also studied. The following sections describe the analysis in details.

\subsection{Influence of number of conflicting vehicles $\left(N_{s}\right)$ on service delay}

Conflicts at uncontrolled intersections generate when both turning and conflicting vehicles arrive at the same time stamp. Thus, the conflicting flow rate corresponding to turning vehicles can be expressed in the form of Eq. (2) which states that number of conflicting vehicles $\left(N_{S}\right)$ is directly proportional to the conflicting flow rate but is inversely proportional to the $T_{i}-T_{1}$. Conversely, $T_{i}-T_{1}$

\footnotetext{
$\overline{4}$ Table S3 provided along with Supplementary Material 1.
} 
Table 1 Service delay $\left(D_{\text {service }}\right)$ and number of conflicting vehicle $\left(N_{s}\right)$ relations

\begin{tabular}{lll}
\hline Vehicle class & Model obtained & $\begin{array}{l}\text { Co-efficient of } \\
\text { determination } \\
\left(R^{2}\right)\end{array}$ \\
\hline Cars & $D_{\text {service }}=4.067 * \exp ^{0.056 . N_{s}}$ & 0.789 \\
SUVs & $D_{\text {service }}=4.384 * \exp ^{0.054 * N_{s}}$ & 0.8163 \\
Two-wheelers & $D_{\text {service }}=2.092 * \exp ^{0.039 * N_{s}}$ & 0.931 \\
Three-wheelers & $D_{\text {service }}=3.033 * \exp ^{0.062 * N_{s}}$ & 0.892 \\
Light commercial vehicles & $D_{\text {service }}=2.974 * \exp ^{0.052 * N_{s}}$ & 0.742 \\
Heavy commercial vehicles & $D_{\text {service }}=5.231 * \exp ^{0.045 * N_{s}}$ & 0.834 \\
\hline
\end{tabular}

$D_{\text {service }}$ in seconds/vehicle and $N_{S}$ is the number of conflicting vehicles is directly proportional to $N_{\mathrm{s}}$. Often during peak periods, the turning drivers under mixed traffic reject more number of smaller headways and therefore $\left(T_{i}-T_{1}=\right.$ service delay $)$ increases. Thus, with increase in the number of opposing/ conflicting vehicles, the conflicting flow rate remains unaltered. Table 1 shows certain numerical relations between $D_{\text {service }}$ and $N_{\mathrm{S}}$ for all vehicle classes.

On the contrary, during off-peak periods, when volume is low, due to unavailability of shorter gaps, waiting time at the stop line increases. Instantaneous conflicting flow rate does not give appropriate representation of turning behaviour. Thus, the conflicting flow rate suggested by Eq. (1) can be revised to Eq. (2).

Conflicting flow rate $=\frac{N_{S}}{\left(T_{i}-T_{S}\right)+\left(T_{S}-T_{1}\right)}=\frac{N_{S}}{\left(T_{i}-T_{S}\right)+D_{\text {service }}}$

Equation (2) shows that service delay $\left(D_{\text {service }}\right)$ is definitely dependent upon the conflicting flow rate. The error created due to effect of conflicting flow rate on service delay estimation, determination of the exact number of conflicting vehicles is required. A subject turning (left or right) on arrival at the virtual reference bar can reject shorter gaps (lesser than the critical gap) and utilizes a single gap (obviously greater than the critical gap) to finish its turning operation. The arrival distribution of the turning vehicles affects this gap size. In absence of exclusive right or left turning lanes, greater gap rejection takes place for which service delay for turning vehicles increases. This assertion is investigated numerically through the Fig. 2 . Figure 2 represents exponential relation between number of conflicting vehicles $\left(N_{\mathrm{S}}\right)$ and service delay $\left(D_{\text {service }}\right)$ for two-wheelers. Similarly, for all other vehicular classes the relation between conflicting vehicles $\left(N_{S}\right)$ and service delay $\left(D_{\text {service }}\right)$.

\subsection{Conflicting Traffic Flow Effects on Service Delay}

A "power" relation between conflicting traffic flow rate influence on the determination of service delay for vehicle

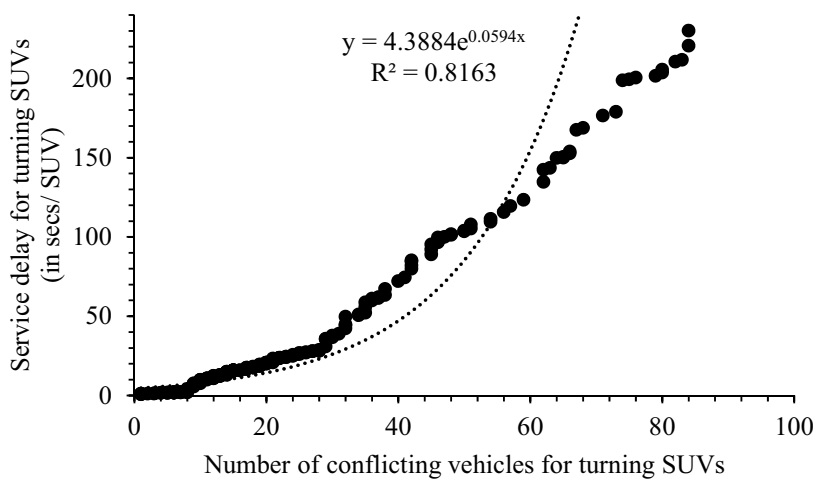

Fig. 2 Service delay Influence on number of conflicting vehicles for SUVs

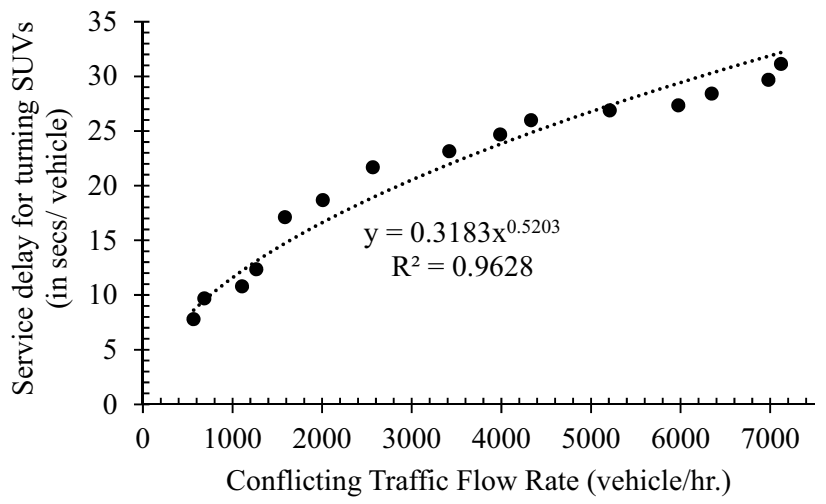

Fig. 3 Conflicting flow rate influences on driver turning service delay for SUVs

category SUVs is shown in Fig. 3. Similarly, for all the other vehicle categories, the same type of relationship, as shown in Table 2.

During peak hours of traffic flow, smaller time gaps (between 1.5 and $2 \mathrm{~s}$ ) are often rejected by non-aggressive drivers, and they accept gaps greater than the critical gap. Therefore, more is the conflicting traffic flow rate, and subject turning vehicles will reject greater number of gaps 
Table 2 Vehicle class wise models representing conflicting traffic flow influence on service delay

\begin{tabular}{lll}
\hline Vehicle category & Model equation & $\begin{array}{l}\text { Co-efficient of } \\
\text { determination } \\
\left(R^{2}\right)\end{array}$ \\
\hline Cars & $D_{\text {service }}=0.4562 * V_{c}^{0.6182}$ & 0.8671 \\
SUVs & $D_{\text {service }}=0.3183 * V_{c}^{0.5203}$ & 0.9628 \\
Two-wheelers & $D_{\text {service }}=0.1316 * V_{c}^{0.4209}$ & 0.9432 \\
Three-wheelers & $D_{\text {service }}=0.3871 * V_{c}^{0.2341}$ & 0.8833 \\
$\begin{array}{l}\text { Light commercial } \\
\text { vehicles }\end{array}$ & $D_{\text {service }}=0.3612 * V_{c}^{0.5624}$ & 0.9532 \\
$\begin{array}{l}\text { Heavy commercial } \\
\text { vehicles }\end{array}$ & $D_{\text {service }}=0.3451 * V_{c}^{0.5129}$ & 0.9732 \\
\hline
\end{tabular}

$D_{\text {service }}$ is in seconds/vehicle and $V_{c}$ is in vehicles/h

before completing the maneuver. A statistical descriptive from service delay estimation is shown in Table S4. ${ }^{5}$

Table S4 (see footnote 5) essentially shows the behavior of service delay for different categories of vehicles, which varies with opposing traffic flow rates. The service delay variation among different vehicle types is due to the variant dynamic and static vehicular characteristics, powerto-weight ratio and driver behavior (driving experience, frequency of driving, familiarity with the intersection, age, gender, etc.). As can be inferred from Table S4 (see footnote 5), the average service delay values for two-wheelers are the least, which are then followed by three-wheelers, cars, SUVs, LCVs, and HCVs. Two-wheelers due to their smaller dimensions and greater acceleration compared to bigger vehicles like 3-W or 4-W accept shorter gaps during turning. Also, 2-W have unique turning behavior for which a lesser turning radius helps them to take every gap presented to them. Also, it can be observed from Table S4 (see footnote 5), 3-W face lesser service delays compared to four-wheeler cars or SUVs. The presence of a single frontal wheel (just like 2-W) and aggressive driver behavior [12] are some of the critical reasons for such behavior. Professional taxis fall under the category of both cars and SUVs, which operate in urban roads of India. Most of these taxis are of the "car" category, with some being "SUV." Therefore, SUV drivers take more caution in accepting smaller gaps than car driving ones, which results in more service delays during turning. Also, the engine capacity of cars varies between 1450 and $1500 \mathrm{cc}$. while that for SUVs is between 2100 and $2450 \mathrm{cc}$. Thus, SUV has more propulsion than cars. Young drivers (22-35 years) mostly drive "Car" rather than SUV. Therefore, driver aggressiveness, along with higher acceleration and lower power-to-weight ratio, compel cars to accept shorter gaps. Light commercial

\footnotetext{
$\overline{5}$ Table S4 is provided along with Supplementary material 1.
}

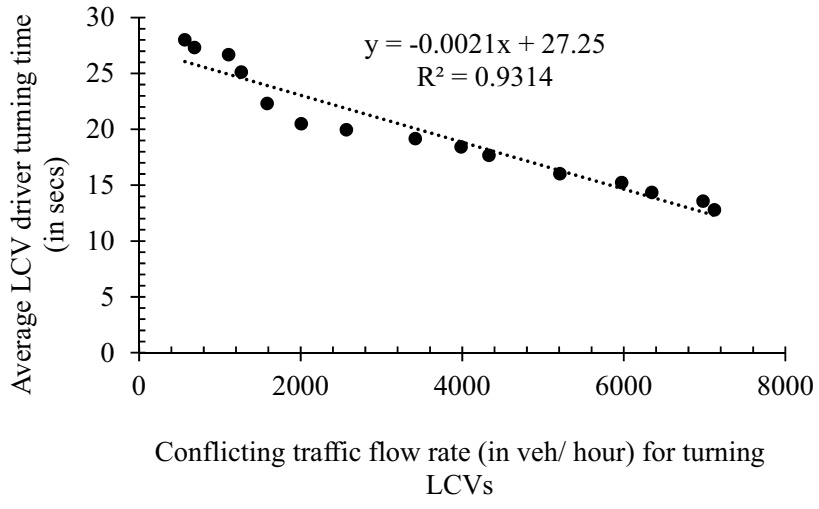

Fig. 4 Average driver turning time (TT) versus conflicting flow rate $\left(V_{c}\right)$ plot for LCVs

vehicles, on the other hand, have a greater length compared to SUVs with lesser engine power-to-weight ratio than HCVs. Thus, the service delay values for LCVs are between SUVs and HCVs.

\subsection{Influence of conflicting traffic flow rate and service delay on driver turning time}

The time required for a right or left turning vehicle to complete its maneuver is assessed in the present study, and all the relevant descriptives are shown in Table S5. ${ }^{6}$ Similar to service delay, the average turning time for two-wheelers is the least followed by threewheelers (3-W), cars, SUV, LCV, and HCV. Two-wheelers, as discussed earlier, have more excellent tractability and lesser frontal dimension compared to other vehicle classes. The influence of average service delay of vehicles $\left(D_{\text {service }}\right)$ on the driver turning time (TT) for light commercial vehicles (LCV) is shown in Fig. 4. Also, three-wheeler drivers in India are the most aggressive, and they continuously interrupt turning trajectories of other vehicles while hooting, leading to lesser turning time. Although such lesser turning often compromises safety and rear-end collisions along with frequent bottleneck formation take place at uncontrolled intersections. Driver behavior related features (like age, gender, or learning experience) make car turning time lesser than SUV. When demand volume at entry increases, gaps become shorter, which in turn increases service delays to vehicles. In such circumstances, when waiting time of turning increases, drivers become impatient and aggressively accept shorter gaps resulting in forced gap acceptance. Forced gap acceptance breaks down the flow of

\footnotetext{
$\overline{6}$ Table S5 provided along with Supplementary material 1.
} 
Table 3 Driver turning time and conflicting flow rate models for vehicular classes

\begin{tabular}{lll}
\hline Vehicle class & Model & $\begin{array}{l}\text { Co-efficient of } \\
\text { determination } \\
\left(R^{2}\right)\end{array}$ \\
\hline Cars & $\Pi T=-0.0015 * V_{c}+21.33$ & 0.8732 \\
SUV & $\Pi \pi=-0.0020 * V_{c}+31.88$ & 0.8933 \\
Two-wheelers & $\Pi \pi=-0.0027 * V_{c}+22.63$ & 0.9722 \\
Three-wheelers & $\Pi \pi=-0.0015 * V_{c}+29.45$ & 0.9022 \\
Light commercial vehicles & $\Pi \pi=-0.0018 * V_{c}+27.25$ & 0.9314 \\
Heavy commercial vehicles & $\Pi T=-0.0022 * V_{c}+25.08$ & 0.8654 \\
\hline
\end{tabular}

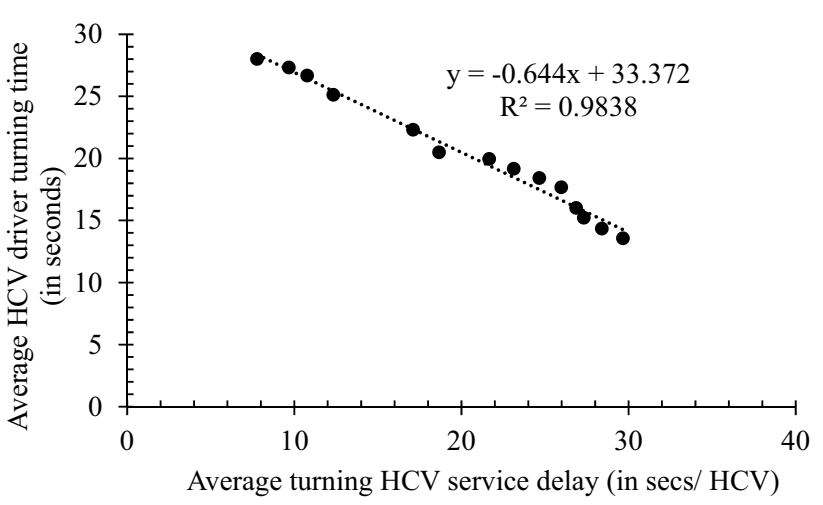

Fig. 5 Influence of service delay on driver turning time for HCVs

conflicting vehicles leading to deceleration. Tian et al. [6] and Obaidat and Elayan [14] practically showed that during high intersection flow, drivers tend to accept lesser gaps shorter than the critical gap after they become impatient, waiting too long at the stop line. Numerical relationships between conflicting traffic flow rate $\left(V_{c}\right)$ in vehicle/h and the average driver turning time (TT) in seconds for different vehicular modes are shown in Table 3.

Figure 5 shows the service delay- driver turning time relationship for HCV as studied.

Table 4 shows service delay ( $\left.D_{\text {service}}\right)$ influence on driver turning time (TT) at the selected uncontrolled intersections considering the six-vehicle classes.

\section{Multi-modal service delay and queue length estimation based on additive conflict flow technique}

An organized method for assessment of delays for various traffic flow streams at multi-modal uncontrolled intersections having complex interactions is presented in this section. For our dataset, intersection no. 4 (fourlegged) and 7 (three-legged) are such sites that have shared public transit -PT (BRTS) lines with parallel vehicular traffic. Intersection 4 and 7 also have sufficiently high volumes of pedestrian movements (although dedicated pedestrian crossings are not present) on the major roads near to the PT stops on both the approaches. Analysis of such kinds of intersections means splitting them into parts. The two essential fundamentals are (1) segregation of different modes for easy identification of virtual priority regulations; (2) demarcation of mode direction. As per the urban mobility plan of India, public transits (especially BRTS) have movement priority over smaller vehicles like cars/SUVs because of their slower dynamic abilities. Conversely, such rules are seldom followed, and thus in many situations, all traffic streams have equal priority under mixed traffic. Therefore, firstly clear regulations regarding modal preference need to be identified. Secondly, it is necessary to split traffic streams as conflicting (C) and non-conflicting (NC) sharing the same space and time within the intersection influence area. It is evident that two non-conflicting streams would seldom come in each other's influence when they move
Table 4 Developed service delay and merging time relations for uncontrolled intersections

\begin{tabular}{lll}
\hline Vehicle modes & Model equations & $\begin{array}{l}\text { Coefficient of } \\
\text { determination } \\
\left(R^{2}\right)\end{array}$ \\
\hline Cars & $\Pi T=-0.535 . D_{\text {service }}+30.551$ & 0.921 \\
SUV & $\Pi T=-0.576 . D_{\text {service }}+30.011$ & 0.939 \\
Two-wheelers & $\Pi T=-0.302 . D_{\text {service }}+23.132$ & 0.877 \\
Three-wheelers & $\Pi T=-0.598 . D_{\text {service }}+27.664$ & 0.793 \\
Light commercial vehicles & $\pi=-0.582 . D_{\text {service }}+25.112$ & 0.955 \\
Heavy commercial vehicles & $\pi=-0.644 . D_{\text {service }}+33.374$ & 0.984 \\
\hline
\end{tabular}


parallel to each other. But, two conflicting streams will compete for space and time. Therefore, to summarize, every intersection can have parallel (NC) and perpendicular (C) movements irrespective of the geometry. The authors would like to recommend four simple steps of analyzing complex, uncontrolled intersections.

1. Development of effective conflict capacity relating to stream interaction behavior.

2. Conflict capacity equation modification factor determination based on priority interaction.

3. Computation of final conflict capacity for every traffic stream

4. Traffic stream service delay calculation

5. Computation of queue length for minor and major road queuing

Step 1 Development of effective conflict capacity relating to stream interaction behavior

A comprehensive algorithm is developed for the development of the conflict capacity equation, as shown in Fig. 6. According to the algorithm depicted through Fig. 6, two types of possibilities arise for the subject stream (k), i.e., (a) $k$ is not affected by any conflicting stream $\mathrm{C} ;(\mathrm{b}) \mathrm{k}$ is affected by any conflicting stream $\mathrm{C}$ but is not in a position to have higher priority over any nonconflicting NC stream. Therefore, in such situations, the effective capacity of the subject stream, $C_{k}$ is obtained as per Eq. (3), which represents the PT and pedestrian movements in Fig. 7.

$C_{k}=S F_{k} X \gamma_{k}$

According to Fig. 7, the PT line and SUV stream are NC, while both are $C$ with the pedestrian movement. Now, as per Indian priority rules, PT has the highest priority (number 1), which is followed by pedestrian movement (number 2) trailed by SUV stream (number 3 ).

In Eq. (3), $\mathrm{SF}_{\mathrm{k}}$ is the saturation flow rate (in vehicles/h) for, $k$, stream and $\gamma_{k}$ is a multiplicative factor used for modification of effective capacity of $k$ movement depending upon the flow ratio. Secondly, when the subject stream, $k$, is affected by any conflicting stream $C$, then $k$ may have higher priority over at least one nonconflicting movement, NC.

This means that NC will have an advantage over $C$, and $C$, in turn, will have priority over $k$. Then, the effective capacity of the subject $k$ movement is obtained using Eq. (4).

$C_{k}=S F_{k} X \gamma_{k}+S F_{k} X$ maximum $\underset{\text { for allN } C_{H P}}{F R_{N C_{H P}} X\left(1-\gamma_{k}\right)}$

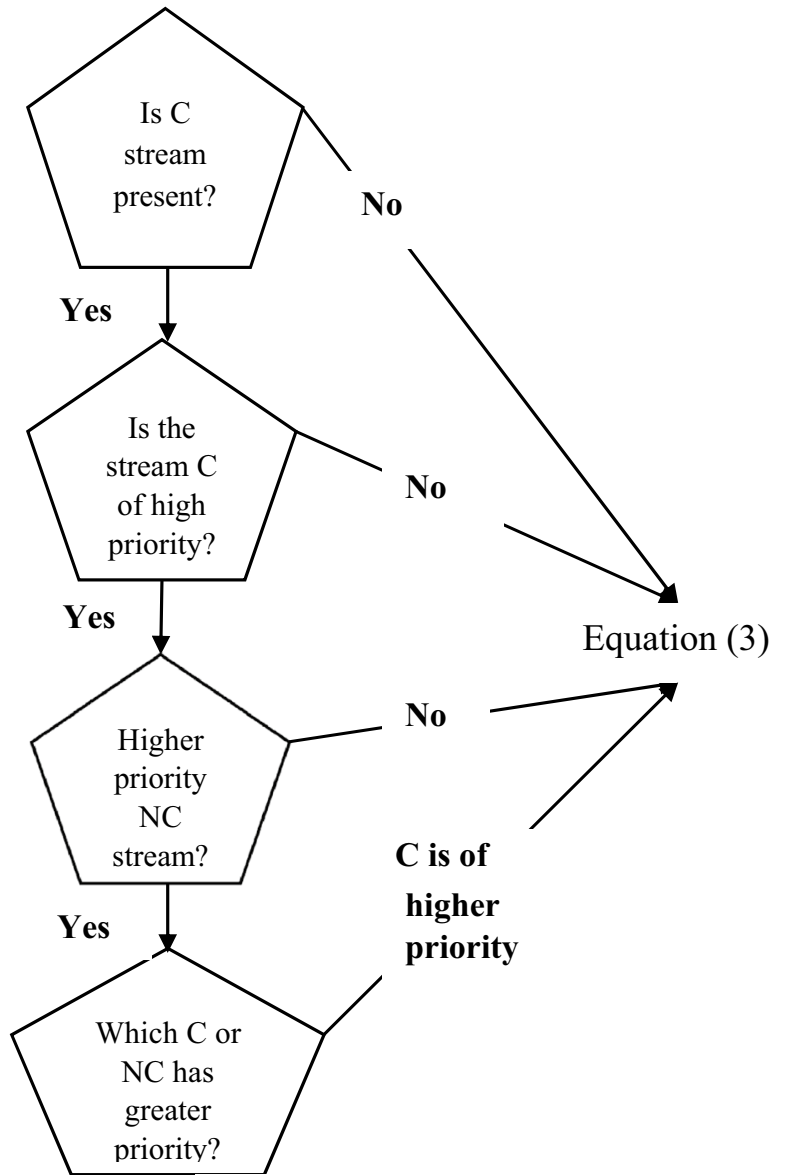

$\leftarrow \begin{aligned} & \text { NC has high } \\ & \text { priority over } \mathrm{C}\end{aligned} \rightarrow$ Equation (4)

Fig. 6 Flow process followed for effective capacity estimation based on modal priority

Pedestrian (number 2)

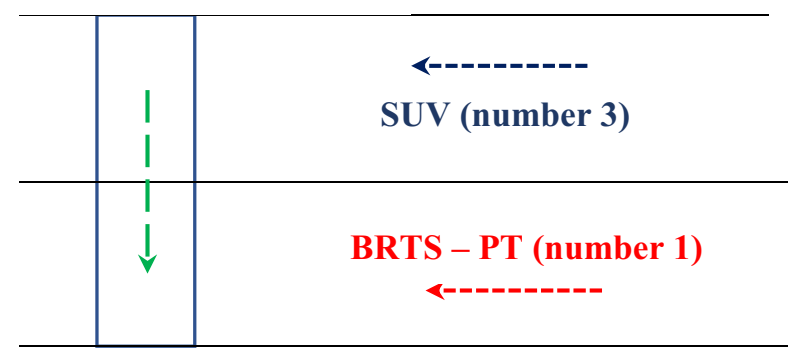

Fig. 7 Indian priority-based conflicting (C) and non-conflicting (NC) traffic streams 


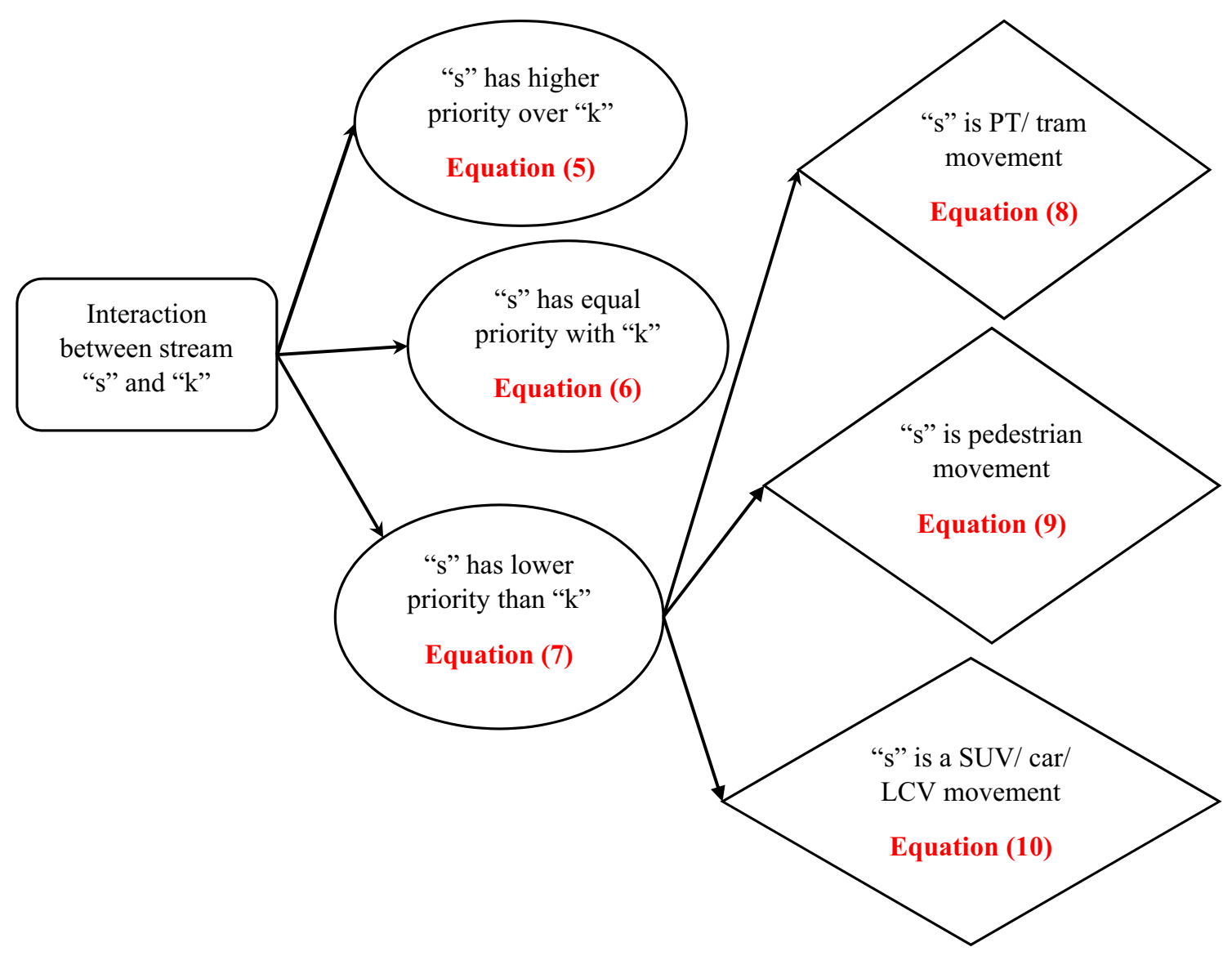

Fig. 8 Determination of conflict capacity modification factor $\gamma_{k}$

In Eq. (4), $N C_{H P}$ represents all non-conflicting streams, $\mathrm{NC}$ having higher priority over $\mathrm{C}$ and $\mathrm{k} ; F R_{N C_{H P}}$ is the flow ratio of all non-conflicting movements having higher ranks other $C$ streams. An example will be the SUV stream in Fig. 7. Flow ratio as per Brilon and Wu [29] and Brilon [30] is a ratio between demand volume $\left(V_{D}\right)$ and the saturation flow $(S F)$ i.e. $\left(F R=V_{D} / S F\right)$. Equation (4) is developed for balanced and understurated traffic flow conditions and so the value of SF is less than equal to 1 but not more than 1 .

Step 2 Determination of the conflict capacity modification factor based on priority interaction

For all conflicting movements $C$ interacting with the subject stream $k$, this step is mandatory. It is well known that at complex uncontrolled intersections in which a lot of directional movements consisting of various modes persist, it is crucial to determine the effective conflicting capacity of the subjecte stream based on the conflicting flow movements individually and then combine to generate effects. Any stream, say, s, that is to be analyzed, requires modification for all $\mathrm{C}$ movements it encounters. In the present study, the modification factor $\gamma_{s k}$ for any movement $s$ interacting with subject movement $\mathrm{k}$ is determined. Figure 8 shows the process flow for determination of the conflict capacity modification factor $\gamma_{k}$, for finding influence of conflicting movement, s, over k. Now, according to the proposed flow process for determination of modification factor, the ultimate value of $\gamma_{k^{\prime}}$ for our subjecte stream " $k$ " interacting with "s" is estimated using Eq. (5).

$\gamma_{k}=\prod_{\text {for all s conflcting with } k} \gamma_{s k}$

In Fig. 6, if the stream of interest i.e. " $k$ " is PT (BRTS) movement and the conflicting stream $C$ i.e. "s" be pedestrian movement, then $\gamma_{k}$ for PT stream should be 1 as " $s$ " has higher priority over " $k$ ". Thus,

$\gamma_{s k}=1$

It is well known that under mixed traffic conditions, two or more movements (involving different modes) can share equal priority. In such cases, the modification factor is obtained as the ratios of individual movements flow ratios (FR). Thus, the case " $s$ " share same priority with " $k$ ", the modification factor is obtained using Eq. (7). 
$\gamma_{s k}=\frac{F R_{s}}{F R_{s}+F R_{k}}$

It is well known that public transits or mass transits operate on a regular basis. Therefore, the saturation flow at intersections will be partly affected by their operation only. Now, if we consider PT (BRTS) movement as " $k$ " and " $s$ " as pedestrian movement. Then, the remaining time in percentage for which the section of intersection can have non-PT movements is $\left(1-\mathrm{SF}_{\mathrm{s}}\right)$. The capacity modification factor for "s" being PT movement with lower priority is estimated from Eq. (8).

$\gamma_{s k}=\left(1-F R_{s}\right)$

Again, in case lower priority movement "s" is pedestrian traffic, then the modification factor is obtained using Eq. (9).

$\gamma_{s k}=\left(1-F R_{s}\right)^{3}$

Alternatively, if "s" being a lower priority movement consists of SUV/car/LCV, the effective conflict capacity modification factor is estimated using Eq. (10).

$\gamma_{s k}=\left(1-F R_{s}\right)^{2}$

Step 3 Computation of final conflict capacity for every traffic stream

The value of $\gamma_{k}$ as obtained from Step 2, is substituted in the Eqs. (3) and (4) of Step 1, the effective conflict capacity of every movement i.e. $C_{k}$ can be obtained.

Step 4 Traffic stream service delay calculation

The total delay equation given in [1] is modified and transformed to "service delay" estimation as represented in Eq. (11).

$D_{\text {total }}=\frac{3600}{C_{k}}-3.5+900 . t\left[(U-1)+\sqrt{(U-1)^{2}+\frac{8 . U}{t . C_{k}}}\right]$

In Eq. (11), $t$ is the analysis time period for data collection which is $15 \mathrm{~min}$ for our study and thus, $t=0.25 \mathrm{~h}$. Also, $U=$ utilization factor $=V_{D} / C_{k}$ for which $V_{D}$ is the movement demand volume is vehicles/h and $C_{k}$ is the effective conflict capacity obtained from Step 3 . Thus, the service delay corresponds to $3600 / C_{k}$ minus the move-up-time in seconds. Also, in Eq. (11), an additional delay of $5 \mathrm{~s}$ is removed as because we are analyzing an uncontrolled intersections and not AWSC (all-way stop controlled). Now as per Indian conditions, the average move-up-time is found to be $3.5 \mathrm{~s}$ (which is $2 \mathrm{~s}$ for AWSC in US conditions) for priority movements. Therefore service delay is given by Eq. (12).
$D_{\text {service }}=\frac{3600}{C_{k}}-3.5$

Step 5 Computation of queue length for minor and major road queuing

TRB $[1,17]$ suggests determination of 95th percentile queue length $\left(Q L_{95}\right)$ for AWSC (all way stop controlled intersections) using the Eq. (13).

$Q L_{95}=225 \cdot \frac{C_{k}}{3600} \cdot\left[(U-1)+\sqrt{(U-1)^{2}+\frac{96 \cdot U}{C_{k}}}\right]$

For the present study, the maximum movement queue length (i.e. $L_{k}$ ) is adopted from Little [31] formula in terms of number of vehicles. Thus, the maximum movement queue length is given by Eq. (14).

$L_{k}=\frac{D_{\text {service }}}{16 / C_{k}}$

\subsection{Application of the conflict technique based service delay estimation}

Figure 9a, b shows line diagram of the typical intersections with priority (in parenthesis) and demand volume per hour.

Type-I intersection is abstracted for two SUV movements (S1 and S2), two pedestrian moevments (PS1, PS2) with a single PT movement. Type-II intersection is abstracted to have four SUV movements (S1, S2, S3 and S4), two pedestrian (PS1, PS2), one LCV and a single PT movement as shown in Fig. 9b. Therefore, the steps shown in the methodology for service delay and queue length estimation is followed for both the cases. The methodology developed is empirically tested for two types of intersections namely-Type I (three-legged Y-shaped) and Type II (four-legged) with shared public transit (i.e. BRTS) lines from the cities of Vashi (Navi Mumbai) and Bhopal (Madhya Pradesh). Both the intersections are multi-modal (i.e. BRTS, SUV, cars, Pedestrians) and multiple conflicting (C) and non-conflicting (NC) streams cross each other. The origin-destination matrices for the Type-I and Type-II intersections is shown Table $56^{7}$ and Table S7. ${ }^{8}$ The SUV demand volumes are taken from this matrices.

\subsection{Analysis of Type-I intersection}

Step 1 The effective capacity, $C_{k}$ of the two SUV streams is determined following the process depicted in Fig. 8. Now,

\footnotetext{
7 Table S6 is provided along with Supplementary material 1.

8 Table S7 is provided along with Supplementary material 1.
} 


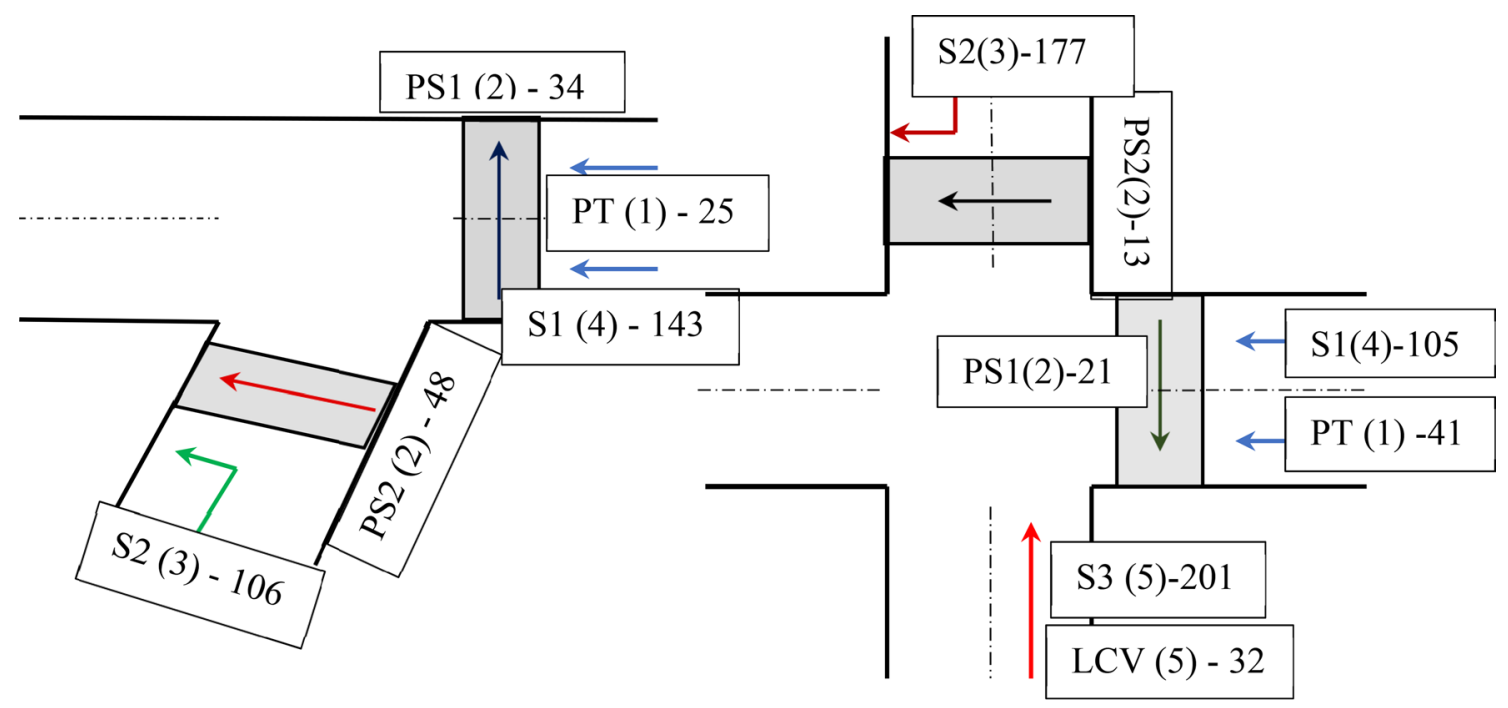

(a)

(b)

Fig. 9 Virtual priority abstract for a Type-I and b Type-/l showing demand volumes of SUVs, LCV, BRTS and pedestrian movements

looking closely, S1 is conflicting with S2 (having higher priority). Whereas, its non-conflicting (NC) stream, PS2 has priority over S2. Thus, S1 can have advantage over S2. Thus,

$C_{S 1}=S F_{S U V} x \gamma_{S 1}+S F_{S U V} x F R_{R 2} x\left(1-\gamma_{R 1}\right)$

S2 also has a conflicting stream with higher priority i.e. PS2 but it does not have any NC stream with high priority than PT. Hence,

$C_{S 2}=S F_{S U V} x \gamma_{S 2}$

Step 2 The capacity modification factor $\left(\gamma_{k}\right)$ is determined for every SUV stream as per flow chart shown in Fig. 9. The three conflicting streams for S1 are PS1 ans S2. Alternatively, S2 has higher priority over S for which $\gamma_{s k}$ is lesser than 1. Thus,

$\gamma_{S 1}=\left(1-F R_{P S 2}\right)^{3} \times\left(1-F R_{S 2}\right)^{3}$

PS2 is the only higher priority C stream to S2. Also, S2 does not cross conflict with PT line. Thus, $\gamma$ for $\mathrm{S} 2$ is given in Eq. (18).

$\gamma_{S 2}=\left(1-F R_{P S 2}\right)^{3}$

Step 3 The steps 1, 2 and 3 are combined together to obtain the effective comflict capacity of the two SUV streams as follows:

$$
\begin{aligned}
C_{S 1}= & S F_{S U V} x\left\{\left(1-F R_{P S 2}\right)^{3} x\left(1-F R_{S 2}\right)^{3}\right\} \\
& +S F_{S U V} x F R_{P S 2} x\left[1-\left\{\left(1-F R_{P S 2}\right)^{3} x\left(1-F R_{S 2}\right)^{3}\right\}\right]
\end{aligned}
$$

$$
C_{S 2}=S F_{S U V} X\left(1-F R_{P S 1}\right)^{3}
$$

\subsection{Analysis of Type -II intersection}

Figure $10 a, b$ shows a comparison between the field estimated and empirically conflict technique based service delay $\left(D_{\text {service }}\right)$ and the queue length $\left(L_{k}\right)$ for all movement streams. The comparison shows that conflict technique based service delay and queue length for the different streams at the multi-modal intersections are very much identical.

The effective conflicting capacity for SUVstreams S1 and S2 for Type-Il intersection is same as given by Eqs. (19) and (20). Additionally, the SUV stream S3 has an effective capacity as determined from Eq. (21) given below.

$$
\begin{aligned}
C_{S 3}= & S F_{S U V} x\left[\left(1-F R_{P T}\right) x\left(1-F R_{S 1}\right)^{3} x\left(1-F R_{P S 2}\right)^{3}\right] \\
& +S F_{S U V} x \max \left(F R_{P S 1}, F R_{S 2}\right) x \\
& \left\{1-\left[\left(1-F R_{P T}\right) x\left(1-F R_{S 1}\right)^{3} x\left(1-F R_{P S 2}\right)^{3}\right]\right\}
\end{aligned}
$$

Also from Fig. 11, it can be observed that it is possible to calculate service delay within $3.2 \mathrm{~s} /$ vehicle/approach of field estimated values.

A maximum percentage error of $40 \%$ for queue length determination shows a maximum sensitivity of $4 \mathrm{~m}$ as observed from Fig. 12. Therefore, the conflict technique based service delay and queue length estimation is really effective under mixed traffic flow. Highest error associated with service delay prediction was found for stream 
Fig. 10 Comparison between average field and empirical a service delay; $\mathbf{b}$ queue length for all movement streams for all thirteen intersections

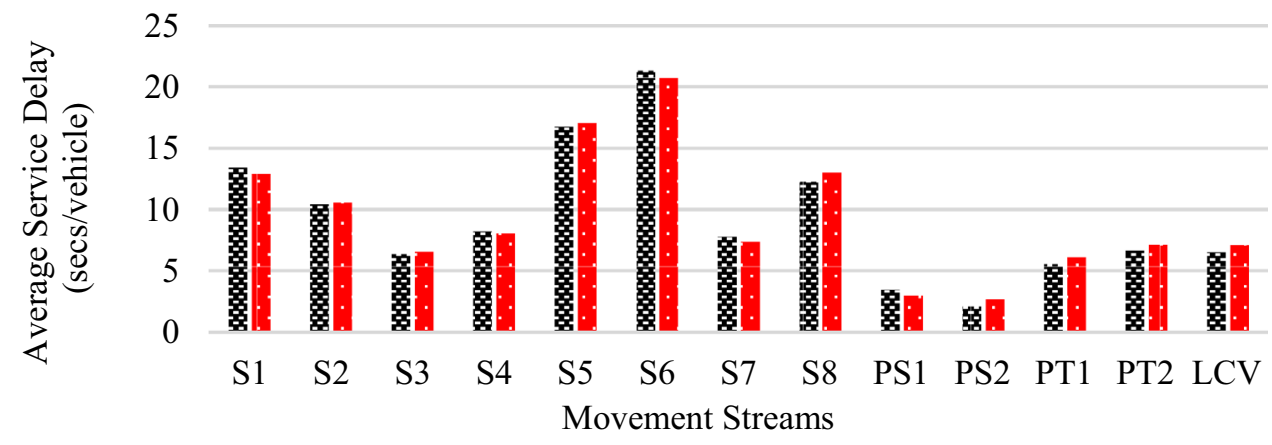

Field estimated SD Empirical calculated SD

(a)

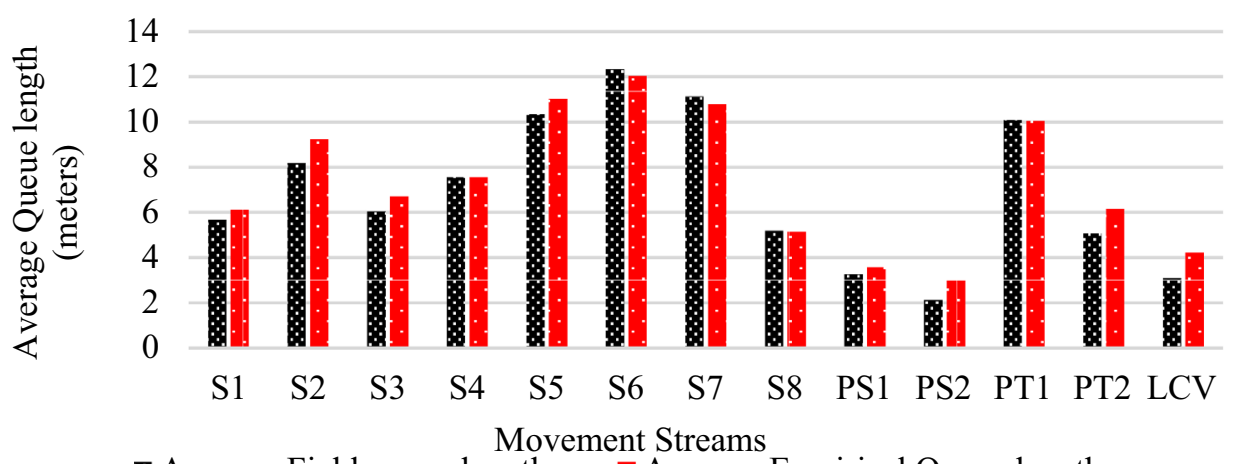

Average Field queue length Average Empirical Queue length

(b)

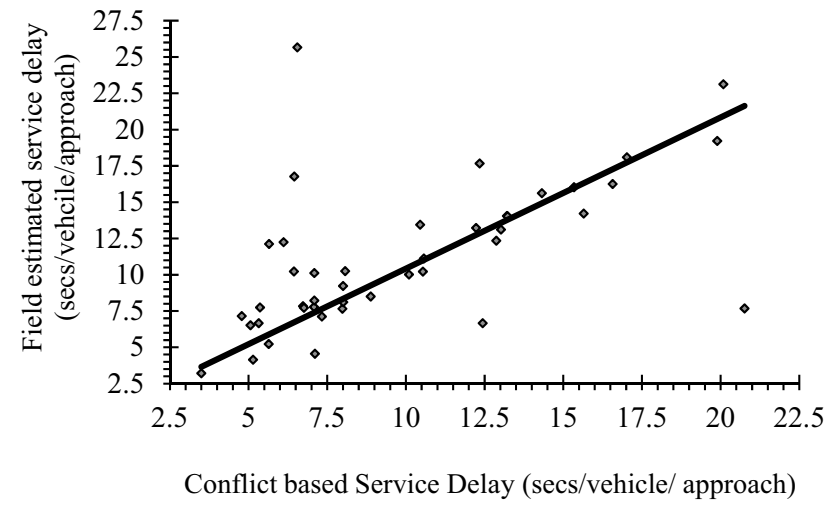

Fig. 11 Scatter diagram for field estimated versus conflict technique based service delay for all the 44 turning movements at the 13 selected uncontrolled intersections

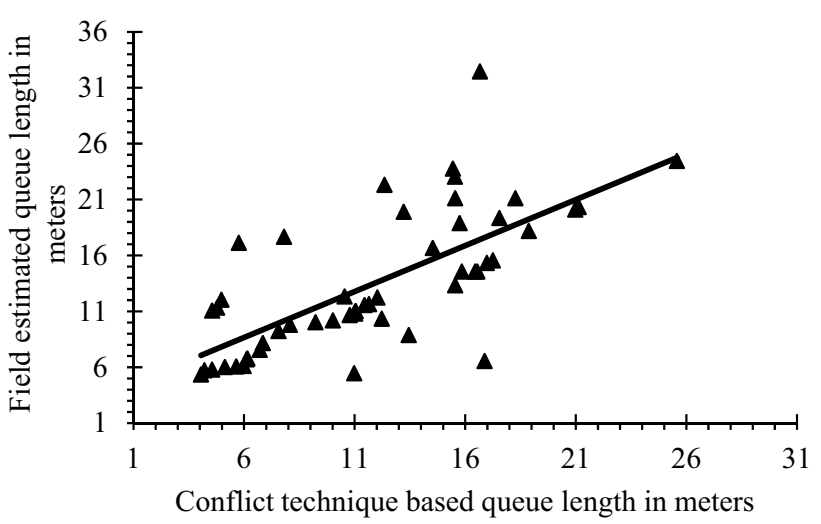

Fig. 12 Scatter diagram showing field estimated versus conflict technique based queue length for all 50 movements at the selected intersections 
Table 5 Average field estimated and calculated service delay and queue length for the three SUV streams for Type-I and Type-II intersections

\begin{tabular}{|c|c|c|c|c|c|c|c|c|c|c|c|c|}
\hline \multirow[t]{2}{*}{$\begin{array}{l}\text { Level of } \\
\text { priority }\end{array}$} & \multirow[t]{2}{*}{ Stream } & \multirow[t]{2}{*}{ Volume (veh/h) } & \multirow[t]{2}{*}{ SF (veh/h) } & \multirow[t]{2}{*}{$C_{\mathrm{k}}(\mathrm{veh} / \mathrm{h})$} & \multicolumn{2}{|l|}{ Field } & \multicolumn{2}{|c|}{ Calculated } & \multicolumn{2}{|c|}{$\begin{array}{l}\text { Percentage error } \\
\text { (\%) }\end{array}$} & \multicolumn{2}{|c|}{ Difference } \\
\hline & & & & & $D_{\text {service }}$ & $L_{k}$ & $D_{\text {service }}$ & $L_{k}$ & $D_{\text {service }}$ & $L_{k}$ & $D_{\text {service }}$ & $L_{k}$ \\
\hline 3 & $\mathrm{~S} 2$ & 327 & 1565 & 1076 & 10.38 & 7 & 10.01 & 7 & 3.56 & 0 & 0.37 & 0 \\
\hline 4 & S1 & 272 & 1342 & 743 & 13.42 & 4 & 12.01 & 5 & 10.5 & -25 & 1.41 & -1 \\
\hline 5 & S3 & 299 & 1409 & 877 & 6.05 & 10 & 9.21 & 6 & -52 & 40 & -3.2 & 4 \\
\hline
\end{tabular}

S3 (i.e. 52\%) for which the maximum queue length of 4 $\mathrm{m}$ is observed.

\section{Validation through Microscopic Simulation in PARAMICS}

Microsimulation based performance assessment is cheap and highly recommended by wide panel of traffic experts all around the globe and has been proved to be effective under complex driver behavior. Two traffic and geometric scenarios are considered. Scenario I consists of a urban corridor containing two signalized and a single T-intersection (Intersection no. 4). One signalized intersection is in the upstream and the other is downstream. The major and minor roads for the T-intersection had bi-directional traffic. Major road had 6 lanes (divided) while minor road has 2-lanes (undivided). Scenario II consists of a urban corridor containing a single 4-legged skewed intersection (Intersection no. 7) and one signalized intersection on the downstream direction. The major road had 5-lanes (divided) while the minor had 4-lanes (divided) with bidirectional traffic flow. Traffic islands, splitter channels or median refuge areas are absent in both the scenarios. The average values of demad volume, saturation flow rate (SF) and effective conflicting capacity $\left(C_{k}\right)$ for SUV streams (S1, S2 and S3) for Type-I and Type-II intersections as shown in Table 5. The percentage error in service delay and queue length comparisons between field estimated and conflict technique based approaches. A maximum percentage error of $52 \%$ for service delay determination shows a maximum sensitivity of $3.2 \mathrm{~s}$.

The mean lane width considered was $3.87 \mathrm{~m}$ and average speed restriction of $40 \mathrm{KpH}$ is considered. Hourly traffic volume data for six vehicle classes are fed into the $\mathrm{O}-\mathrm{D}$ vehicle route assignment module of PARAMICS. Consequently, two 2-h volume data (9:00 AM-11:00 AM for morning; 6:30 PM-8:30 PM for evening) are used. Vehicle count detectors are placed on the individual lanes of the major of minor road turning points of the two uncontrolled intersections under Scenarion I and II. Maximum capacity of 900 vehicles per hour (veh/h) and $1200 \mathrm{veh} / \mathrm{h}$ for minor and major roads is considered. Volume to capacity ratio ranges between $0.5-1.52$ and $0.6-1.75$ is considered for minor and major roads respectively. Intersections are calibrated geometrically (i.e. number of lanes, lane width, gradient, etc.), priority-based (fully uncontrolled, partially uncontrolled, yield controlled, etc.) and gap acceptance parameters.

The PARAMICS car-following and lane-changing model was modified with respect to safe stopping distance, headways and braking distances for all the six vehicle classes. Priority movement knowledge to drivers are removed from the lane changing model and lane changing was allowed for drivers accepting a gap. As can be observed from Fig. 13a, b, the simulated hourly flow rates for right turns are quite similar to the field observations for both the scenarios. It is clear, that, for both morning and evening peak periods, calibration was done efficiently based on the comparison.

Figures $13 a, b]^{9}$ and $14 a, b^{10}$ represents the PARAMICS simulation results for hourly major right turn entry demand volume and mean queue length for all the 13 intersections based on Scenario-I and II. Scenario-I (IT1, IT3, IT4, IT6, IT7, IT10, IT13) is concerned with performance analysis of three-legged T-type uncontrolled intersections with fixed time signals in its proximity (i.e. 500 to $700 \mathrm{~m}$ ) both in the upstream and downstream ends.

As validated from Fig. $15 a, b,{ }^{11}$ the conflict technique based queue length and service delay method formulated in the present study is very effective under both the scenarios for all the thirteen selected sites for both morning and evening peak periods. Similar observations were found for minor road queue size under both the scenarios. Traffic characteristics including six vehicle types, static vehicle dimensions (length, width and height), dynamic vehicle characteristics (maximum, minimum and desired speed, accelerations and decelerations) and traffic

\footnotetext{
${ }_{9}$ Values are taken from Table S8 provided along with Supplementary material 1.

${ }^{10}$ Values are taken from Table S8 provided along with Supplementary material.

11 Values are taken from last and second last columns of Table S8 provided along with Supplementary material 1.
} 
Snro-I: Morning peak (9:3010:30AM)

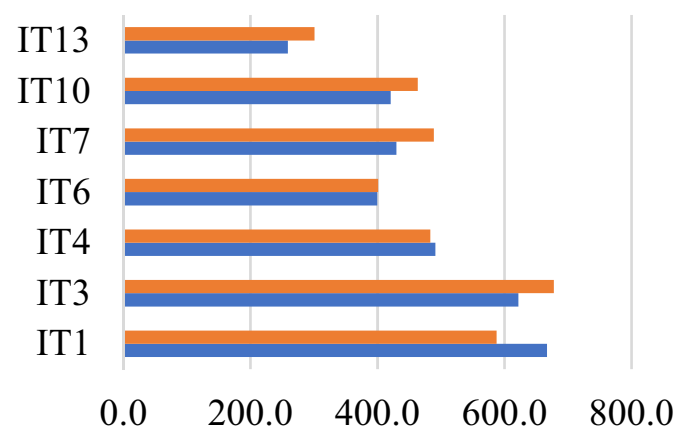

- Field hourly right turn flow

- Simulated hourly right turn flow
Snro-I: Evening peak (6:30-7:30

PM)

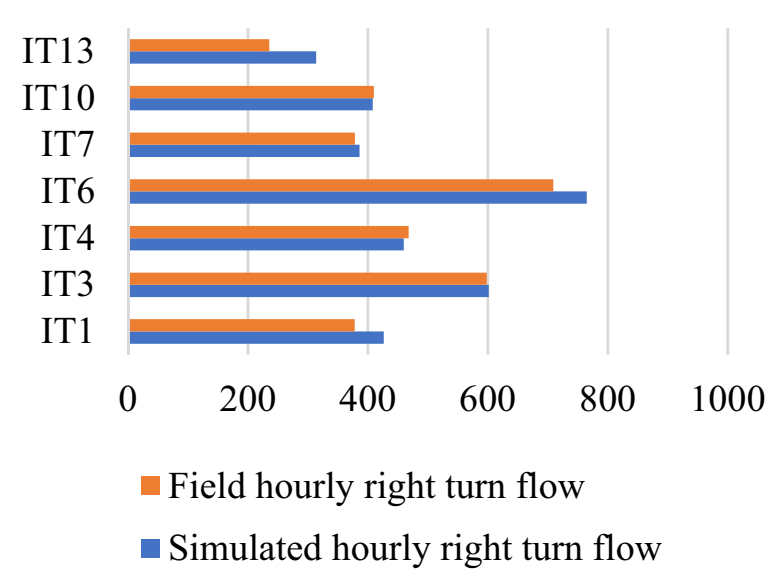

(a) Scenario - I

\section{Snro-II: Morning peak (9.30 - 10:30 AM)}

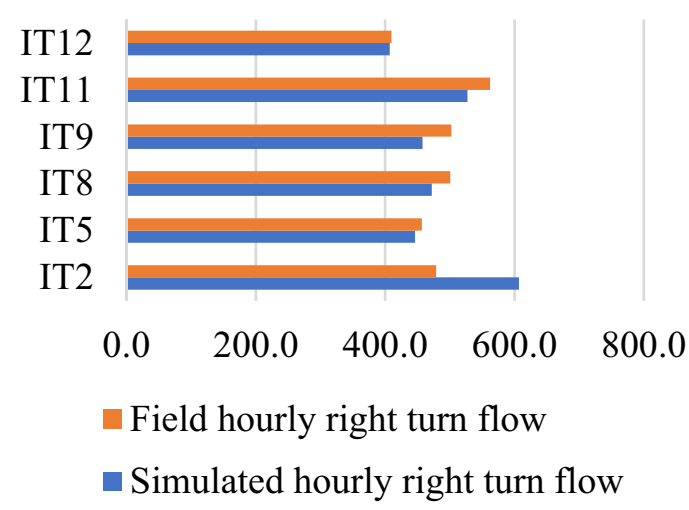

Snro-II: Evening peak(6:30-7:30 PM)

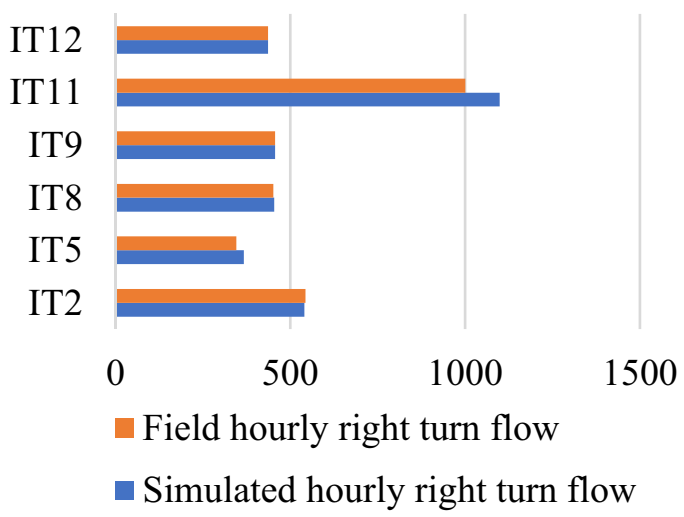

(b) Scenario - II

Fig. 13 Simulated and field estimated morning and evening peak hourly flow rates for right turns (vehicles/h/lane) under a Scenario-l; b Scenario-II

composition were calibrated for Scenario I and II. A rigorous scrutiny between the field inputs and simulation outputs was performed along with traffic modelling for the calibration. Statistical tolerance using the GEH statistic is utilized after completing several hours of simulation runs as depicted by Eq. (22).

$G E H=\sqrt{\frac{2(S-F)^{2}}{S+F}}$
In Eq. (22), GEH is Geoffrey E. Havers statistic which represents acceptance standards in travel demand for casting models, $\mathrm{S}$ is the simulated hourly demand traffic flow rate $(v e h / h)$ amd $F$ is the field estimated hourly traffic flow rate (veh/h). The GEH statistic for the 13 intersections was estimated to be lesser than 4 which means that simulated output and field inputs are significantly same. Scenario-II (IT2, IT5, IT8, IT9, IT11, IT12) is concerned with analysis of 4-legged uncontrolled intersections with advese queuing effects from upstream fixed time signals. Both the scenarios are representation of 


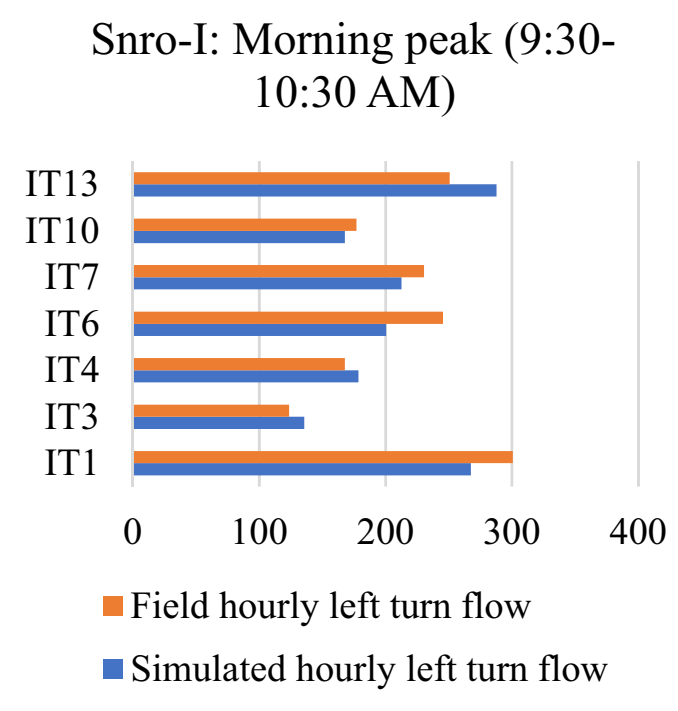

Snro-I: Evening peak (6:30-7:30

PM)

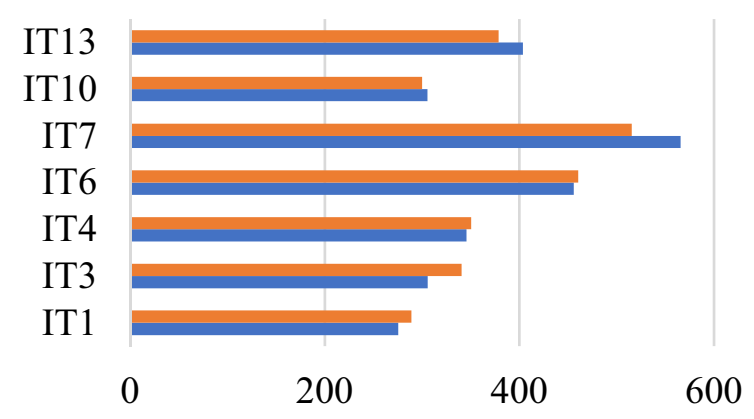

- Field hourly left turn flow

- Simulated hourly left turn flow

(a) Scenario-I
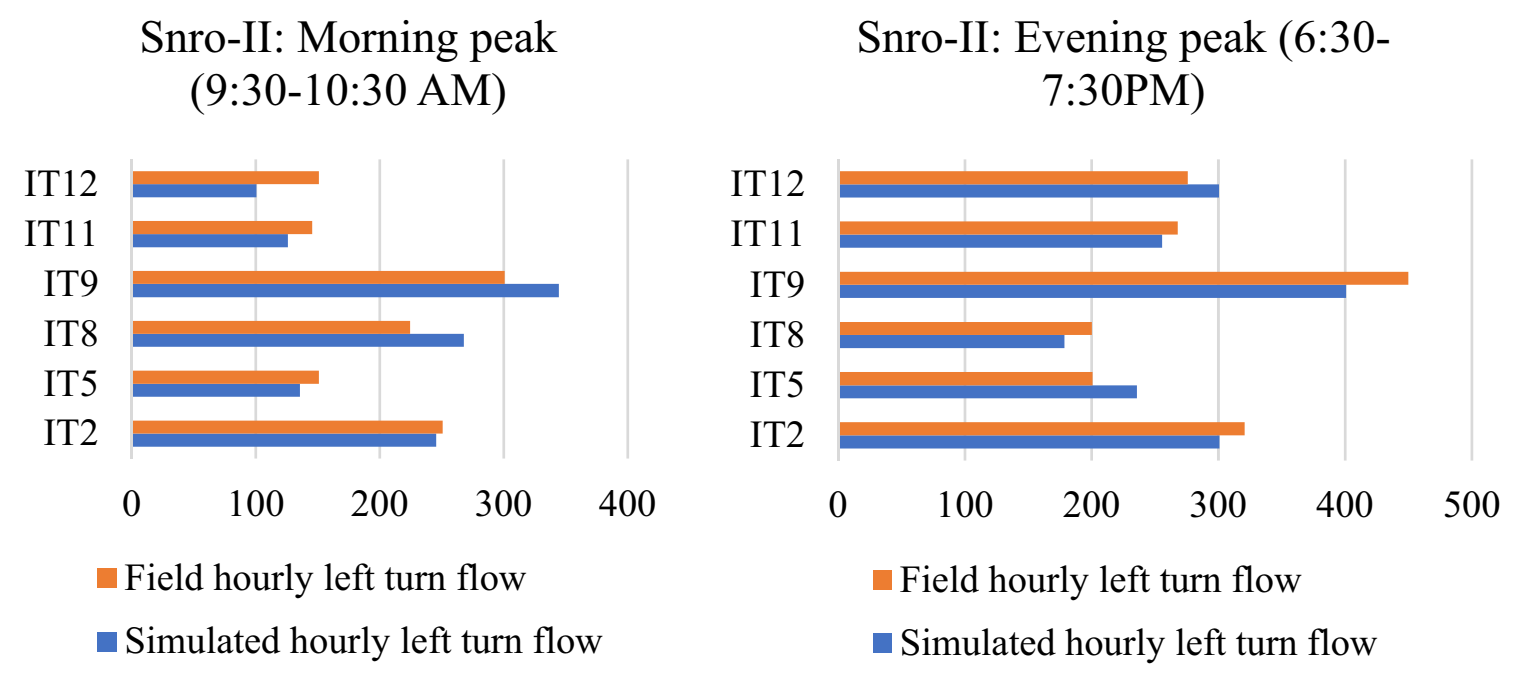

(b) Scenario-II

Fig. 14 Simulated and field estimated morning and evening peak hourly flow rates for left turns (vehicles/h/lane) under a Scenario-l; b Scenario-II

real traffic situations as observed during data collection. Figure $14 a, b$ indicates close compliance of the simulated hourly left turn flow rates with the field estimated values inferring better calibration with field geometric and traffic conditions at the thirteen different sites corresponding to Scenario-I and II. Fig. $\mathrm{S} 2^{12}$ and Fig. $S 3^{13}$ shows the visualization screenshots for the two scenarios (Snro-I and Snro-II) involving Type-I (3-legged) and Type-II (4-legged) intersections in the Quadstone PARAMICS (v. 6.3.0.1) graphical user interface (GUI).

\footnotetext{
12 Fig. S2 is provided along with Supplementary material 2.

${ }^{13}$ Fig. S3 is provided along with Supplementary material 2.
} 
Snro-I: Morning peak (9:30 10:30 AM)

IT13
IT10
IT7
IT6
IT4
IT3
IT1
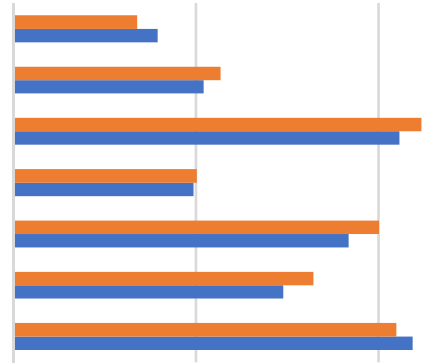

0.0

10.0

20.0

30.0

- Conflict technique based major road avg. queue length

- Simulated major raod avg. queue length
Snro-I: Evening peak (6:30-7:30

PM)

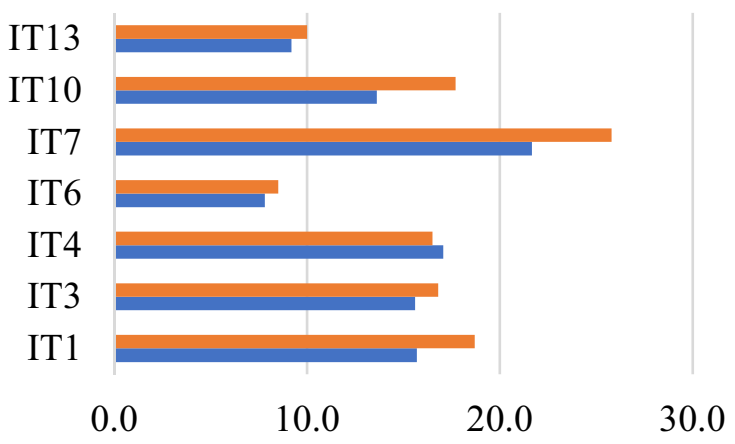

(a) Scenario-I

Snro-II: Morning peak (9:3010:30 AM)

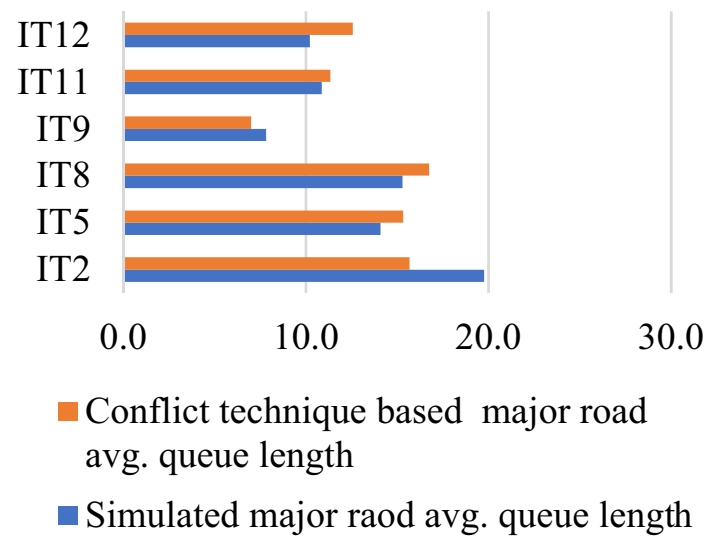

Snro-II: Evening peak (6:307:30 PM)

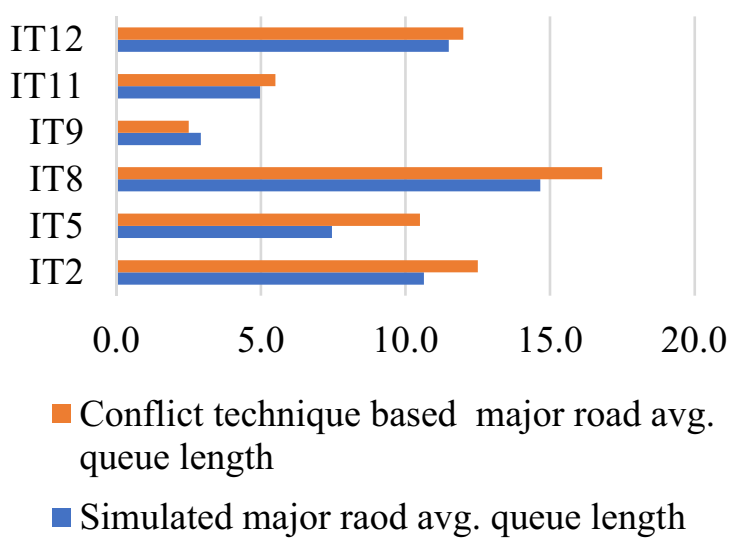

(b) Scenario-II

Fig. 15 Simulated and field estimated conflict based queue length (in meters) during morning and evening peak hours for a Scenario-l; b Scenario-II

\section{Statistical analysis governing significance of the service delay, driver turning time and queue length modelling using PARAMICS}

Statistical modelling is essential to predict expected service delay and queue length for minor/major roads at intersections based on number of conflicting vehicles
$\left(N_{S}\right)$, peak hourly turning volume $\left(V_{D}\right)$ and peak hourly conflicting flow rate $\left(V_{C}\right)$ as explanatory variables. Generally, poisson distribution is followed during delay and queue length modeling but under mixed and non-lane based conditions it may lead to over-dispersion and under-estimation. Assuming a poisson process, if $X_{j}$ be the peak hourly service delay counts i.e. the random variable, mean of poisson distribution i.e. $\mathrm{E}\left(X_{j}\right)=\omega_{j}$ is equal to the variance i.e. $\operatorname{Var}\left(X_{j}\right)=\omega_{j}$, where, $\omega_{j}$ is the expected 
Table 6 Negative exponential model results with and without mock variable M (i.e. NE and NEM) under average expected service delay values during peak hour

\begin{tabular}{|c|c|c|c|c|c|c|c|c|}
\hline \multirow[t]{2}{*}{ Parameters } & \multicolumn{4}{|l|}{ NE } & \multicolumn{4}{|l|}{ NEM } \\
\hline & Estimate & SE & LRT value & AME & Estimate & SE & LRT value & AME \\
\hline Intercept & 0.20451 & 0.2213 & 6.7832 & - & -1.3456 & 0.7513 & 4.981 & - \\
\hline$N_{S}$ & 0.43421 & 0.8144 & 2.8761 & 3.5422 & 0.5321 & 0.4912 & 3.418 & 2.875 \\
\hline$V_{D} / 1000$ & 0.32574 & 0.1563 & 41.235 & 6.7432 & 1.6702 & 0.4351 & 30.98 & 11.892 \\
\hline$V_{C} / 1000$ & 0.77325 & 0.2039 & 9.0345 & 7.6321 & 1.70239 & 0.5619 & 11.32 & 14.629 \\
\hline $\begin{array}{l}M\left(1 \text { for } V_{C} \leq 1000 \text { veh } / \mathrm{h} \text { and } 0\right. \\
\left.\text { for } V_{C}>1000\right)\end{array}$ & - & - & - & - & 1.78456 & 0.57032 & 12.089 & - \\
\hline overdispersion & 0.43590 & & & & 0.28705 & & & \\
\hline$O$ & 65 & & & & 65 & & & \\
\hline$L L(\beta)$ & -135.7624 & & & & -131.4562 & & & \\
\hline$L L(0)$ & -154.3207 & & & & -149.7654 & & & \\
\hline$\rho^{2}$-value & 0.12315 & & & & 0.16134 & & & \\
\hline$\chi^{2}=-2[L L(0)-L L(\beta)]$ & 42.6752 & & & & 51.3427 & & & \\
\hline$\chi^{2}=-2\left[L L\left(\beta_{N E}\right)-L L\left(\beta_{N E M}\right)\right.$ & 14.0876 & & & & & & & \\
\hline$A / C$ & 309.45218 & & & & 277.5545 & & & \\
\hline$B I C$ & 315.76334 & & & & 304.3122 & & & \\
\hline
\end{tabular}

$S E$ standard error, $L R T$ likelihood ratio test, $M$ mock/dummy variable, $N E$ negative exponential model, $N E M$ negative exponential model with mock variable, $M, A M E$ mean marginal effects

values of service delay. Thus, the negative exponential (NE) model is given in Eq. (23).

$g\left(x_{j}\right)=1 / \beta \cdot e^{-\frac{\left(x_{j}-\omega_{j}\right)}{\beta}}$

where, $\mu$ denotes the location parameter and $\beta$ denotes the scale parameter $\forall, x_{j} \geq \omega_{j}$ and $\beta>0$. Now, let us consider, $z$, to be a vector having $N$ number of co-variate and $a$ be a vector having $N$ (unknown) co-efficient. Now for a poisson process and negative exponential relation, the expected service delay regression model is stated as $\vartheta_{j}=g\left(z_{j}: \delta\right)$, where, $g($.$) is a function. Thus, the regression$ model used in the study is governed by Eq. (24).

$\vartheta_{j}=\exp \left(z_{j}^{T} \delta\right)$

In order to obtain the estimates, $\hat{\delta}$ and $\hat{\beta}$ for example, of unknown parameters $\delta$ and $\beta$, log-likelihood function under the governing model is to be maximized. Often maximum likelihood (ML) shows invariance towards estimation. In this study, $\hat{\vartheta}_{j}=\gamma_{j}: \hat{\delta}$, is the ML estimate for expected service delay/queue lengths at peak hour. Log-likelihood is negative integer and Greene [32] suggested to use the likelihood ratio test (LRT) as a measure for goodness-of-fit in regression. LRT is widely used to evaluate two competing models. LRT also provides evidence regarding sensitivity of models and their limitations towards removal of explanatory parameters.
The goodness-of-fit statistics related to the parameter restrictions in the established regression models is measures using the Chi square $\left(\chi^{2}\right)$ statistic give by Eq. (25).

$\chi^{2}=-2[L L(\delta)-L L(0)]$

In Eq. (25), $L L(\delta)$ is the log-likelihood for full model, $L L(0)$ is log-likelihood for constant-only model. $\chi^{2}$-statistic is $\chi^{2}$ distributed with 1 degrees of freedom (DOF). Influence of additional co-variate in full model can be analyzed using LRT using the $\rho^{2}$-statistic which also analyses goodness-offit and is calculated as per Eq. (26).

$\rho^{2}=1-L L(\delta) / L L(0)$

As suggested by Washington et al. [33], $\rho^{2}$-value lies between 0 and 1 and higher values denote lesser model invariance. The Akaike information criteria (AIC) and the Bayesian information criteria (BIC) as calculated using Eqs. (27) and (28) is also used. Lesser values of AIC and BIC denotes reliable model.

$A I C=2 . N-2 . L L(\delta)$

$B I C=N \cdot \ln (O)-2 \cdot L L(\delta)$

In Eqs. (27) and (28), $N$ is the number of explanatory variables including the dispersion parameter in the full model and $O$, denotes the total number of observations. The authors calculated marginal effects $(M E)$ to quantify 
Table 7 Negative exponential model results with and without mock variable, $M$ (i.e., NE and NEM) under average expected turning time values during peak hour

\begin{tabular}{|c|c|c|c|c|c|c|c|c|}
\hline \multirow[t]{2}{*}{ Parameters } & \multicolumn{4}{|l|}{$\mathrm{NE}$} & \multicolumn{4}{|l|}{ NEM } \\
\hline & Estimate & SE & LRT value & AME & Estimate & SE & LRT value & AME \\
\hline Intercept & 0.03425 & 0.4109 & 0.01875 & - & -1.2513 & 1.321 & 9.083 & - \\
\hline$N_{S}$ & 0.55421 & 1.09873 & 1.40321 & 7.9654 & 0.6217 & 0.512 & 3.05 & 6.2314 \\
\hline$V_{D} / 1000$ & 0.68355 & 0.26197 & 17.5034 & 3.0092 & 0.0943 & 0.805 & 22.851 & 17.604 \\
\hline$V_{C} / 1000$ & 1.04325 & 0.28074 & 19.0993 & 4.89251 & -0.3425 & 0.30917 & 12.033 & 19.0834 \\
\hline $\begin{array}{l}M\left(1 \text { for } V_{C} \leq 1000 \mathrm{veh} / \mathrm{h} \text { and } 0\right. \\
\left.\text { for } V_{C}>1000\right)\end{array}$ & - & - & - & - & 3.09871 & 0.3509 & 9.4567 & - \\
\hline overdispersion & 1.6653 & & & & 1.0512 & & & \\
\hline 0 & 45 & & & & 45 & & & \\
\hline$L L(\beta)$ & -144.3253 & & & & -107.6214 & & & \\
\hline LL(0) & -158.9876 & & & & -133.0899 & & & \\
\hline$\rho^{2}$-value & 0.07431 & & & & 0.11066 & & & \\
\hline$\chi^{2}=-2[L L(0)-L L(\beta)]$ & 17.68426 & & & & 25.06471 & & & \\
\hline$\chi^{2}=-2\left[L L\left(\beta_{N E}\right)-L L\left(\beta_{N E M}\right)\right.$ & 10.9844 & & & & & & & \\
\hline$A / C$ & 256.7621 & & & & 270.093 & & & \\
\hline$B I C$ & 281.3055 & & & & 281.655 & & & \\
\hline
\end{tabular}

$S E$ standard error, $L R T$ likelihood ratio test, $M$ mock/dummy variable, $N E$ negative exponential model, $N E M$ negative exponential model with mock variable, $M, A M E$ average marginal effects

independent variable impact on the expected dependent variable value. $M E$ reflects one unit change in $z_{j}$ impact on the modelled variable, $\vartheta_{j}$ and is governed by Eq. (29). In the equation, $K$ is the number of explanatory parameters added in the full model.

$M E_{z_{j N}}^{\gamma_{j}}=\frac{\partial \vartheta_{j}\left(x_{j}=K\right)}{\partial z_{j N}}$

\subsection{Results from negative exponential model with equations}

Table 6 shows that expected signs for co-efficient are obtained for the negative exponential (NE) model. Service delay is positively related to the peak hourly turning flow, number of conflicting vehicles and also with conflicting flow rate. As inferred from Table 6, the NE model with mock variable, $M$ has greater efficiency towards service delay prediction because of high values of loglikelihood, better $\rho^{2}$-value and minimum values for $A I C$ and BIC. Over-dispersion $\left(=1 / \omega_{j}\right)$ is lower for the NE model compared to NEM. At $10 \%$ significance levels, as per the LRT (> 2.87), all parameters considered are sstatistically significant. Also, it can be observed that coefficients for $V_{c}$ are greater than that of $V_{D}$ indicating service delay for turning maneuvers is affected more by conflicting flow during peak hours. The claim has direct connection with reduction in time gaps and thus longer waiting times leading to greater service delay. Again if demand volume is kept constant, then arrival of greater conflicting vehicles might result in extensive queue developments. Average marginal effects due to $V_{C}$ is found to be higher than $V_{D}$ and $N_{S}$. This shows that service delay is greatly affected by $V_{C}$. Table 7 shows the estimation results for the NE and NEM models under average driver turning time peak hourly values. Table 7 also shows that turning time $(T T)$ is positively related to $V_{D}$ and $N_{S}$ but negatively related to $V_{C}$. However, the NEM model showed greater efficiency than the NE model because of higher values of log-likelihood (LL), $\rho^{2}$-value with lesser $A I C$ and $B I C$ values. Now, in this case, $N_{S}$ has higher co-efficient than $V_{C}$ and $V_{D}$ indicating driver turning time (TT) is mostly affected due to number of conflicting vehicles. This can also be validated with higher average marginal effects of $N_{S}$ than $V_{D}$ or $V_{C}$. Table 8 shows that queue length $\left(L_{\mathrm{k}}\right)$ is positively associated with $N_{S}, V_{D}$ and $V_{C}$. In this case, the mock variable, $M$ was not found significant at $10 \%$ level and thus is removed during modelling. Also, as inferred from Table 8, the co-efficient corresponding to turning demand volume $\left(V_{D}\right)$ is more than conflicting flow rate $\left(V_{C}\right)$ and number of conflicting vehicles $\left(N_{\mathrm{S}}\right)$. This assures that queue formatations are mostly due to high turning approach volume $\left(V_{D}\right)$ rather than higher $N_{S}$ or $V_{C}$ values. The predictive models used for determination of mean values of service delay (in s), driver turning time (in s) and queue length (in meters) during peak hourly flow at uncontrolled 
Table 8 Negative exponential model results under average expected queue length values during peak hour

\begin{tabular}{llllll}
\hline Parameters & \multicolumn{2}{l}{ NE } & & & \multicolumn{2}{l}{ Model parameters } \\
\cline { 2 - 5 } & Estimate & SE & LRT value & AME & \\
\hline Intercept & 0.50092 & 0.175032 & 15.3421 & - & - \\
$N_{S}$ & 0.26537 & 0.310125 & 35.9081 & 17.6833 & - \\
$V_{D} / 1000$ & 0.67663 & 0.38692 & 36.0845 & 23.1468 & - \\
$V_{C} / 1000$ & 0.44311 & 0.15809 & 20.02214 & 6.55134 & - \\
Overdispersion & - & - & - & - & 0.28541 \\
$O$ & - & - & - & - & 60 \\
$L L(\beta)$ & - & - & - & - & -154.3021 \\
$L L(0)$ & - & - & - & - & -193.2457 \\
$\rho^{2}$-value & - & - & - & - & 0.1106355 \\
$\chi^{2}=-2[L L(0)-L L(\beta)]$ & - & - & - & - & 43.10902 \\
$A I C$ & - & - & - & - & 305.432 \\
$B I C$ & - & - & - & - & 345.788 \\
\hline
\end{tabular}

SE standard error, $L R T$ likelihood ratio test, NE negative exponential model, $A M E$ mean marginal effects

Table 9 Importance of model explanatory inputs according to sensitivity analysis

\begin{tabular}{|c|c|c|c|c|}
\hline $\begin{array}{l}\text { Uncontrolled intersection } \\
\text { performance measure }\end{array}$ & Attribute & $\begin{array}{l}\text { Number of conflicting } \\
\text { vehicles- } N_{S}\end{array}$ & $\begin{array}{l}\text { Turning demand volume in vehi- } \\
\text { cles per hour per lane- } V_{D}\end{array}$ & $\begin{array}{l}\text { Conflicting flow rate in } \\
\text { vehicles per hour per } \\
\text { lane- } V_{C}\end{array}$ \\
\hline \multirow[t]{2}{*}{ Service delay $\left(D_{\text {service }}\right)$} & $S_{j}(\%)$ & 35.45 & 13.76 & 18.42 \\
\hline & Rank & 1 st & $3 r d$ & $2 n d$ \\
\hline \multirow[t]{2}{*}{ Driver turning time $(T T)$} & $S_{j}(\%)$ & 19.18 & 14.15 & 40.57 \\
\hline & Rank & 2nd & $3 r d$ & $1 \mathrm{st}$ \\
\hline \multirow[t]{2}{*}{ Queue length $\left(L_{k}\right)$} & $S_{j}(\%)$ & 16.17 & 37.92 & 8.54 \\
\hline & Rank & 2nd & 1st & $3 r d$ \\
\hline
\end{tabular}

intersections is portrayed by Eqs. (30), (31) and (32). A mock variable, $M$ is considered to account for the marginal effects during modelling which considers values of 1 for $V_{c} \leq 1000$ veh/he and 0 otherwise.

$D_{\text {service }}=e^{\left[-1.3456+\left(0.5321 . N_{S}\right)+1.6702 \cdot\left(\frac{v_{D}}{1000}\right)+1.70239 .\left(\frac{v_{C}}{1000}\right)\right]}$

$\pi=e^{\left[-1.2513+\left(0.6217 . N_{S}\right)+0.0943 \cdot\left(\frac{v_{D}}{1000}\right)-0.3425 \cdot\left(\frac{v_{C}}{1000}\right)\right]}$

$L_{k}=e^{\left[0.50092+\left(0.26537 . N_{S}\right)+0.67663 \cdot\left(\frac{V_{D}}{1000}\right)+0.44311 \cdot\left(\frac{v_{C}}{1000}\right)\right]}$

The model equations given by Eqs. (30), (31) and (32) are obtained based on the PARAMICS simulation results obtained after calibration of all the thirteen uncontrolled intersections.

Sensitivity analysis for finding important explanatory variables affecting service delay, driver turning time and queue length is carried out utilizing the Eqs. (30) and (31) which were then used in the NE model building process.
$S_{j}=\frac{D_{j}}{\sum_{j=1}^{g} D_{j}} X 100$

$D_{j}=V_{\max }\left(y_{j}\right)-V_{\min }\left(y_{j}\right)$

In the Eqs. (33) and (34), $S_{j}$ denotes the sensitivity of a jth explanatory variable expressed in percentage; $D_{j}$ represents the difference between the maximum " $V_{\max }\left(y_{j}\right)$ " and minimum value " $V_{\min }\left(y_{j}\right)$ " predicted jth variable (i.e., service delay, turning time or queue length) which is calculated after substituting the maximum and minimum values of the jth explanatory variable and also the average values of each input variable in the proposed service delay (Eq. 30), driver turning time (Eq. 31) and queue length (Eq. 32) models; $g$ represents the number of explanatory inputs ( 3 in this case). Table 9 shows the sensitivity ratio values in percentage and ranking of the input variables according to their importance in the NE model building process. According to the sensitivity ratio (\%) values 
Table 10 Performance regarding prediction ability of the proposed service delay, driver turning time and queue length models

\begin{tabular}{llllllll}
\hline $\begin{array}{l}\text { Uncontrolled intersection } \\
\text { performance measure }\end{array}$ & Type of dataset & $R^{2}$ & $E$ & RMSE & AAE & MAE & MAPE (\%) \\
\hline Service delay $\left(D_{\text {service }}\right)$ & Training & 0.834 & 0.855 & 0.125 & 0.167 & 0.602 & 3.56 \\
& Validation & 0.902 & 0.865 & 0.321 & 0.128 & 0.587 & 5.87 \\
Driver turning time $(T T)$ & Training & 0.872 & 0.872 & 0.176 & 0.076 & 0.523 & 7.12 \\
& Validation & 0.865 & 0.812 & 0.221 & 0.312 & 0.598 & 6.32 \\
Queue length $\left(L_{k}\right)$ & Training & 0.912 & 0.801 & 0.108 & 0.218 & 0.576 & 4.56 \\
& Validation & 0.933 & 0.892 & 0.207 & 0.277 & 0.591 & 3.78 \\
\hline
\end{tabular}

presented in Table 9, for service delay estimation, the number of conflicting vehicles (with $\mathrm{S}_{\mathrm{j}}=35.45 \%$ ) is the prime input variable. Conflicting flow rate (in vehicles/h/lane) was found to be of prime importance (with $\mathrm{S}_{\mathrm{j}}=40.57 \%$ ) for driver turning time estimation at uncontrolled intersections. On the other hand, turning demand volume (in vehicles/h/lane) is the most important input variable (with $\left.\mathrm{S}_{\mathrm{j}}=37.92 \%\right)$ as far as queue length estimation at uncontrolled intersections.

\subsection{Validation of the Proposed Models}

The prediction accuracy of all the three developed models were also evaluated based on certain statistical attributes like the co-efficient of determination $\left(R^{2}\right)$, Nash-Sutcliffe co-efficient $(E)$, root mean square error (RMSE), average absolute error $(A A E)$, maximum absolute error (MAE), and the mean absolute percentage error (MAPE). All these attributes were derived individually for both training and validation datasets (training-70\% and testing-30\%) based on the field estimated (i.e. conflict technique based) and simulation model outputs as shown in Table 10. Often it can be observed that $R^{2}$ estimates get biased and therefore, the Nash-Sutcliffe estimate $(E)$, which gives an idea of how good are the proposed models is expressing the mean variance in the data. $E$ compares the field estimated values and the simulated outputs as shown in Eq. (35).

$E=\frac{E_{1}-E_{2}}{E_{1}}$

$E_{1}=\sum_{s=1}^{a}\left(F_{j}-\bar{F}_{j}\right)^{2}$

$E_{2}=\sum_{s=1}^{a}\left(F_{j}-S_{j}\right)^{2}$

In the Eqs. (35), (36) and (37), $F_{j}$ denotes the field estimated value (service delay, turning time or queue length); $\bar{F}_{j}$ denotes the mean of the field estimated value (service delay, turning time or queue length); $S_{j}$ denotes the PARAMICS simulation value (service delay, turning time or queue length); $a$ represents the number of intersection approaches utilized for model building (i.e., 45 and 20 for training and validating the model respectively). Table 10 shows that all the three models proposed (based on PARAMICS simulation) are quite strong in terms of prediction ability having higher values of $R^{2}$ and $E$ whereas lower values of RMSE, AAE, MAE and MAPE.

The conceivable mathematical mean given as $\left\{M=\sum_{c=1}^{J} y_{c / j}\right\}$, represents the average of sample measurements for $y_{1}, y_{2}, y_{3}, \ldots, y_{c}$ (conflict technique based or simulated service delay) with $J$ being total number of samples. The estimates based on the predictive models says, modelled $M_{D_{\text {serice }}}=9.672 \mathrm{~s}$ against average conflict technique based value of $9.053 \mathrm{~s}$. Alternatively, $\left(M_{T T}=10.853 \mathrm{~s}\right)$ against field estimated turning time value of $10.541 \mathrm{~s}$. While for average expected minor road queue length, $M_{L_{k}}=8.79$ magainst conflict based average queue length of $9.2 \mathrm{~m}$. The contrast shows reliable levels of agreement between simulated and predicted recital measures.

\section{Conclusions}

Attempts have been devised to assess driver waiting time before turning initiation (i.e. service delay), time required to merge with conflicting flow (i.e. driver turning time) and queue length at uncontrolled multi-modal intersections under complex and diverse traffic scenario. Furthermore, conflict technique based adaptive service delay and queue length under various priority scenarios for every traffic stream have been determined. Data from thirteen uncontrolled intersections from several regions of India are taken for assessment. The errors for average service delay and queue length predictions using conflict technique are found within $3.2 \mathrm{~s} /$ vehicle/approach and 4 $\mathrm{m}$ and even lower for some streams. The conflict based algorithm proposed by the authors can be easily automated and the only inputs are priorities and directions of movements/traffic streams, demand volume, movement saturation flows which are easily retrievable even under 
traffic heterogeneity. It is observed that service delay for a turning vehicle depends highly on number of conflicting vehicles $\left(N_{S}\right)$, conflicting flow rate $\left(V_{C}\right)$ and peak hourly turning demand volume $\left(V_{D}\right)$. According to microscopic analysis based on field observations, driver turning time is linearly affected by turning driver service delay $\left(D_{\text {service }}\right)$ but after simulation it was found the relation was negative exponential (NE). Similarly, negative exponential (NE) relations were observed for driver turning time (TT) and queue length $\left(L_{k}\right)$ based on microscopic simulation. Conversely, closer arithmetic means between simulated and field observed values showed that both kinds of relationship is appropriate. For simplicity, authors deem the micro simulation based relations to be more statistically efficient. Models which are developed under homogeneous and priority-disciplined driver behavioral traffic flow situations can never be used to evaluate performance measures under mixed traffic conditions. Also, such models become infeasible when applied for impatient and inconsistent drivers commonly found in India. Additionally, the capacity and level of service guidelines followed in India [34] evaluates unsignalized intersections on the basis of volume to capacity (v/c) ratio only and does not consider delay as a measure of effectiveness.

The findings from the research will help traffic decision makers to: (1) comparing diverse design substitutes; (2) quickly judge and take counter actions on prevailing intersections ensuring greater mobility; (3) speedy performance assessment and renovation of uncontrolled intersections based on traffic demand and conflicting flow rate variation.

Author's contributions Conceptualization: (Siddhartha Rokade); Methodology: (Sarvesh P.S. Rajput); Formal analysis and investigation: (Suprabeet Datta), (Siddhartha Rokade); Data Extraction and Modulation: (Suprabeet Datta); Writing-original draft preparation: (Suprabeet Datta); Writing - review and editing: (Suprabeet Datta), (Siddhartha Rokade); Resources: (Siddhartha Rokade); Supervision: (Siddhartha Rokade), (Sarvesh P.S. Rajput).

\section{Compliance with ethical standards}

Conflict of interest On behalf of all the authors, the corresponding author wants to declare that there are no competing interests with any other studies exclusive to the research presented in this manuscript.

Availability of data and material The authors have collected data used for analysis, and no other third-party publisher or source is responsible for data reproduction, and some/all data collected/reproduced is available with permissions from the authors of this manuscript.
Code availability All/some data will be made available to the journal on behalf of the authors on request.

\section{References}

1. HCM (2016) A guide for multimodal mobility analysis. Transportation Research Board, Washington. DC, USA. http://www. trb.org/Main/Blurbs/175169.aspx

2. SP 41 (1964) Guidelines for the design of at-grade intersections in rural and urban areas, Indian Roads Congress. Ministry of Road Transport \& Highways (MoRTH), New Delhi, pp 42-103. https://www.scribd.com/doc/265296809/IRC-SP-41-pdf

3. Daganzo CF (1977) Traffic delay at unsignalized intersections: clarification of some issues. Trans Sci 11(2):180-189

4. Kimber R, Summersgill I, Burrow IJ (1986) Delay process at unsignalized junctions: the interrelation between geometric and queueing delay. Trans Res Part B Method 20B(6):457-476. https://doi.org/10.1016/0191-2615(86)90025-1

5. Heidmann D, Wegmann $H$ (1997) Queuing at unsignalized intersections. Trans Res Part B Method 31B(3):239-263. https://doi. org/10.1016/S0191-2615(96)00021-5

6. Tian ZZ, Kyte M, Vandehey M, Kittelson W, Robinson B (2001) Simulation-based study of traffic operation characteristics at allway-stop-controlled intersections. Trans Res Rec 1776(1):75-81. https://doi.org/10.3141/1776-10

7. Lutinnen RT (2004) Capacity and level of service at Finnish unsignalized intersections. Finnish Road Administration, Helsinki

8. Chodur J (2005) Capacity models and parameters for unsignalized intersections in Poland. J Trans Eng Part A Syst 131(12):924-930. https://doi.org/10.1061/ (ASCE)0733-947X(2005)131:12(924)

9. Pitzinger P, Spacek P (2009) [Ein neuer Ansatz zum Abschätzen von Leistungsfähigkeit und Verkehrsablauf an ungesteuerten Knotenpunkten] (In: Polish). Strassenverkehrstechnik, Heft 4. https://www.aramis.admin.ch/Texte/?ProjectID=24705

10. Chandra S, Agrawal A, Ashalatha R (2009) Microscopic analysis of service delay at uncontrolled intersections in mixed traffic conditions. J Trans Eng Part A Syst 135(6):323-329. https://doi. org/10.1061/(ASCE)0733-947X(2009)135:6(323)

11. Ashalatha $R$, Chandra $S$ (2011) Service delay analysis at TWSC intersections through simulation. KSCE J Civ Eng 15(2):413-425. https://doi.org/10.1007/s12205-011-1125-9

12. Kanagaraj V, Srinivasan KK, Sivanandan R (2010) Modelling vehicular merging behaviour under heterogeneous traffic conditions. Trans Res Rec 2188(1):140-147. https://doi. org/10.3141/2188-15

13. Forbush TR (2011) Automated delay estimation at signalized intersections: phase I concept and algorithm development. Master thesis retrieved from. All Thesis and Dissertations, pp 271-302. https://scholarsarchive.byu.edu/etd/2471/

14. Obaidat TA, Elayan MS (2013) Gap acceptance behavior at U-turn median openings-case study of Jordan. Jordan J Civ Eng $7(3): 332-341$

15. Caliendo C (2014) Delay time model at unsignalized intersections. J Trans Eng Part A Syst 140(4):1-13. https://doi. org/10.1061/(ASCE)TE.1943-5436.0000696

16. Menendez M, Guler I, Puffe E (2015) Traffic flow at uncontrolled urban intersections with attention to different modes of traffic: determination of representative standard values and interrelations. Technical report VSS 2011/308, Road Transport Commission Switzerland 
17. HCM (2010) Highway capacity manual. Transportation Research Board, Washington DC, USA. http://www.trb.org/Main/Blurb s/164718.aspx

18. Sil G, Mohapatra SS, Dey PP, Chandra S (2016) Merging process of U-turns at uncontrolled median openings under mixed traffic conditions. Transport 33(2):370-379. https://doi. org/10.3846/16484142.2016.1247295

19. Shatnawi I, Ping Y, Khliefat I (2018) Automated intersection delay estimation using the input-output principle and turning movement data. Int J Trans Sci Technol 7(4):137-150. https://doi. org/10.1016/j.ijtst.2018.04.001

20. Khattak AJ, Jovanis PP (1990) Capacity and delay estimation for priority unsignalized intersections: conceptual and empirical issues. Trans Res Rec 1287:129-137

21. Zongzhong T, Kyte M, Coylar J (1997) Field measurement of capacity and delay at unsignalized intersections. ITE J 67(4):22-26

22. Al-Omari B, Benekohal RF (1999) Hybrid delay models for unsaturated two-way stop-controlled intersections. J Trans Eng Part A Syst 125(4):291-296. https://doi.org/10.1061/ (ASCE)0733-947X(1999)125:4(291)

23. Bonneson JA, Fitts JW (1999) Delay to major street through vehicles at two-way stop-controlled intersections. Trans Res Part A Policy Pract 33(3):237-253

24. Teply S, Abou Henaidy M, Hunt JD (1997) Gap acceptance behavior-aggregate and logic perspective Part I. Traffic Eng Control 38(9):474-482

25. Kyte M, Clemow C, Mahfood N, Lall BK, Khisty CJ (1991) Capacity and delay characteristics of two-way stop-controlled intersections. Trans Res Rec 1320(1):160-167
26. Sun (Jian) D, Elefteriadou L (2010) Research and implementation of lane changing model based on driver behaviour. Trans Res Rec 2161(1):1-10. https://doi.org/10.3141/2161-01

27. Sun(Jian) D, Zhang L, Chen F (2013) Comparative study on simulation performances of CORSIM and VISSIM for urban street network. Simul Model Pract Theory 37(7):18-29. https://doi. org/10.1016/j.simpat.2013.05.007

28. Kittelson WK, Vandehey MA (1991) Delay effects on driver estimation approaches for priority unsignalized intersections: conceptual and empirical issues. Trans Res Rec 1320(1):154-159

29. Brilon W, Wu N (2001) Capacity at unsignalized intersections derived by conflict technique. Trans Res Rec 2167:82-90. https ://doi.org/10.3141/1776-11

30. Brilon W (2008) Delay at unsignalized intersections. Trans Res Rec 2071:98-108. https://doi.org/10.3141/2071-12

31. Little JDC (1961) A proof for the queueing formula: $L=\lambda W$. Oper Res 9(3):383-387. https://doi.org/10.1287/opre.9.3.383

32. Greene W (2007) Limdep, version 9.0. Economic Software, Plainview, New York

33. Washington SP, Karlaftis MG, Mannering FL (2011) Statistical and economic methods of transportation data analysis. Chapman and Hall, CRC Press, Boca Raton

34. CRRI (2017) Indian highway capacity manual. Ministry of Road Transport and Highways (MoRTH). 1st edn. Centre for Scientific and Industrial Research, New Delhi, pp 8-1-8-25. https://trid.trb. org/view/1509422

Publisher's Note Springer Nature remains neutral with regard to jurisdictional claims in published maps and institutional affiliations. 